\title{
Genotypic and Phenotypic Variables Affect Meiotic Cell Cycle Progression, Tumor Ploidy, and Cancer-Associated Mortality in a brca2-Mutant Zebrafish Model
}

\author{
L. Mensah, J. L. Ferguson, and H. R. Shive 1 \\ Department of Population Health and Pathobiology, North Carolina State University, College of Veterinary Medicine, \\ 1060 William Moore Drive, Raleigh, Raleigh, NC, USA \\ Correspondence should be addressed to H. R. Shive; hrshive@ncsu.edu
}

Received 26 November 2018; Revised 10 January 2019; Accepted 28 January 2019; Published 26 February 2019

Guest Editor: Zhihua Kang

Copyright (C) 2019 L. Mensah et al. This is an open access article distributed under the Creative Commons Attribution License, which permits unrestricted use, distribution, and reproduction in any medium, provided the original work is properly cited.

\begin{abstract}
Successful cell replication requires both cell cycle completion and accurate chromosomal segregation. The tumor suppressor BRCA2 is positioned to influence both of these outcomes, and thereby influence genomic integrity, during meiotic and mitotic cell cycles. Accordingly, mutations in BRCA2 induce chromosomal abnormalities and disrupt cell cycle progression in both germ cells and somatic cells. Despite these findings, aneuploidy is not more prevalent in BRCA2-associated versus non-BRCA2-associated human cancers. More puzzlingly, diploidy in BRCA2-associated cancers is a negative prognostic factor, unlike non-BRCA2-associated cancers and many other human cancers. We used a brca2-mutant/tp53-mutant cancer-prone zebrafish model to explore the impact of BRCA2 mutation on cell cycle progression, ploidy, and cancer-associated mortality by performing DNA content/cell cycle analysis on zebrafish germ cells, somatic cells, and cancer cells. First, we determined that combined brca2/tp53 mutations uniquely disrupt meiotic progression. Second, we determined that sex significantly influences ploidy outcome in zebrafish cancers. Third, we determined that brca2 mutation and female sex each significantly reduce survival time in cancer-bearing zebrafish. Finally, we provide evidence to support a link between BRCA2 mutation, tumor diploidy, and poor survival outcome. These outcomes underscore the utility of this model for studying BRCA2-associated genomic aberrations in normal and cancer cells.
\end{abstract}

\section{Introduction}

Generation of cell progeny lies at the heart of virtually all biological processes. Successfully performing this fundamental cell behavior requires both completion of the cell cycle and faithful replication and segregation of chromosomal content. Both meiotic and mitotic cell cycles are governed by these principles, although clear mechanistic differences exist (reviewed by Duro E and Marston AL [1]). If cell cycle progression is disturbed during mitotic or meiotic cell cycles, potential adverse outcomes include cell cycle arrest, chromosomal aberrations, and/or missegregation; the latter outcomes may cause chromosomal instability and aneuploidy.

The tumor suppressor gene BRCA2 functions in multiple pathways that affect both meiotic and mitotic cell cycles, and thereby genomic stability. These include homology-directed repair (HDR), replication fork maintenance, spindle assembly checkpoint (SAC), cytokinesis, and telomere homeostasis (reviewed by Venkitaraman AR [2]). Figure 1(a) indicates phases of the meiotic and mitotic cell cycles during which BRCA2 is known to function and the corresponding cellular DNA content in each phase. In meiosis, BRCA2 functions in prophase I of meiosis I; cells enter meiosis I with 4C DNA content and exit meiosis I with 2C DNA content following the first meiotic division. In mitosis, BRCA2 participates in multiple processes that span from the G2 checkpoint in late G2 phase to cytokinesis in M phase, as described below. Cells enter G2 phase with $4 \mathrm{C}$ DNA content and exit M phase with 2C DNA content.

In mammalian germ cells, loss of functional Brca2 resulted in cell cycle arrest in meiotic prophase I and persistent DNA damage [3, 4]. Additionally, aberrant chromosomal segregation during meiosis was described in brca2-mutant Arabidopsis gametophytes [5]. In primary somatic cells (mouse embryonic fibroblasts), loss of functional Brca2 caused cell cycle arrest and both structural and numerical 


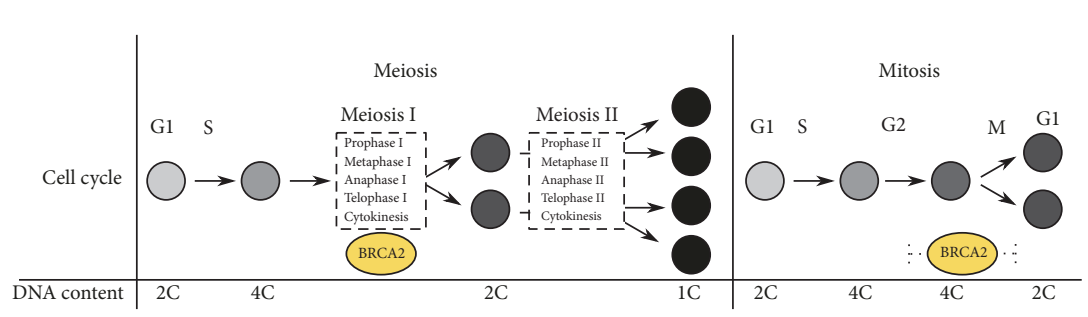

(a)

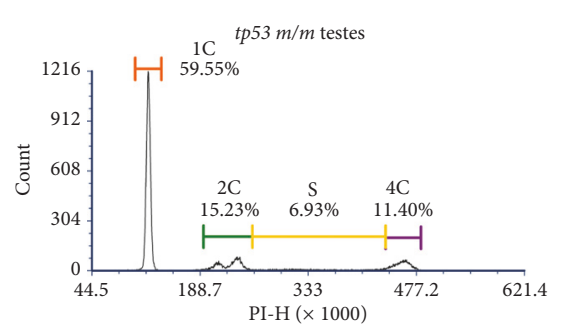

(c)

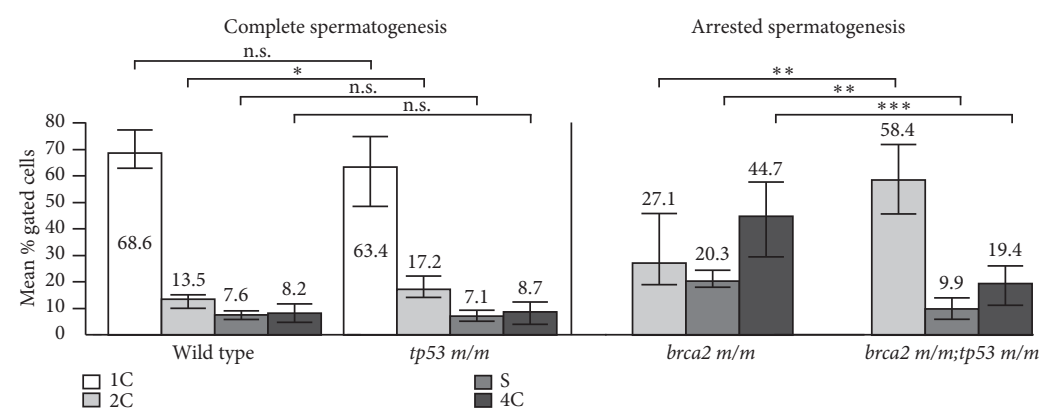

(f)

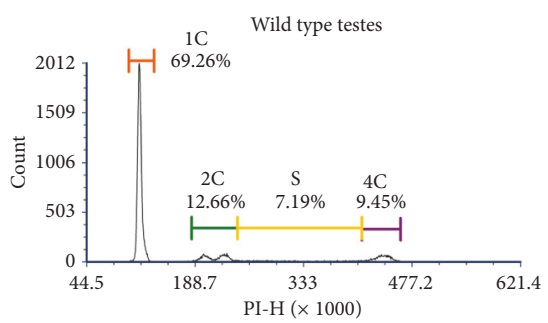

(b)

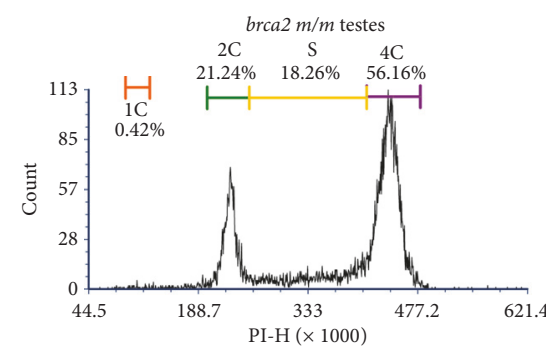

(d)

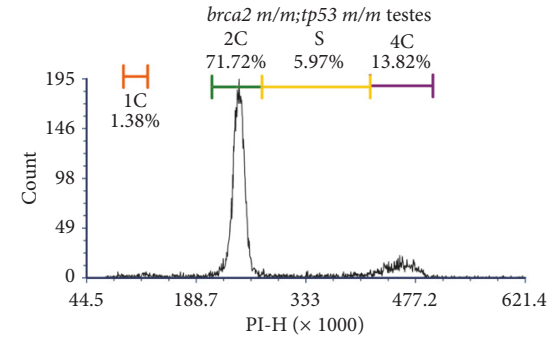

(e)

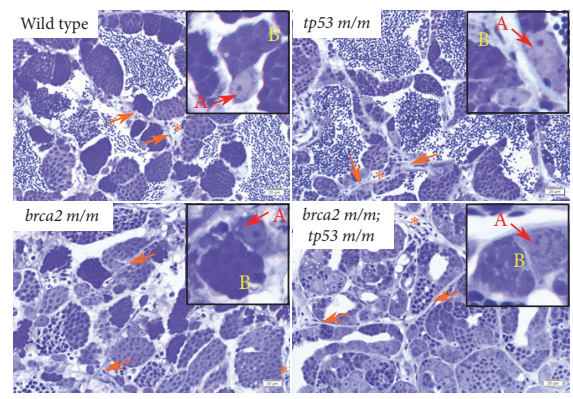

(g)

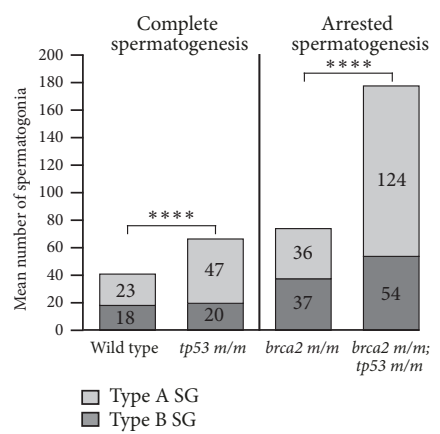

(h)

Figure 1: brca2 and tp53 mutations alter distribution of cells according to DNA content in adult zebrafish testes. (a) Comparison of cell progression through meiosis and mitosis with corresponding DNA content (designated as 1C, 2C, or 4C). BRCA2 participates in DNA repair during prophase I of meiosis I and performs multiple functions between late $\mathrm{G} 2$ and $\mathrm{M}$ phases of mitosis, as indicated by the positions of the yellow ovals. ((b)-(e)) Propidium iodide (PI) fluorescence histograms of testes derived from wild type (b), tp53 m/m (c), brca2 m/m (d), and brca $2 \mathrm{~m} / \mathrm{m} ; \mathrm{tp} 53 \mathrm{~m} / \mathrm{m}$ zebrafish testes (e). (f) Mean percent of gated cells clustered by DNA content for wild type, $t p 53 \mathrm{~m} / \mathrm{m}, \mathrm{brca} 2 \mathrm{~m} / \mathrm{m}$, and brca $2 \mathrm{~m} / \mathrm{m} ; \mathrm{tp} 53 \mathrm{~m} / \mathrm{m}$ zebrafish testes. The mean percent of gated cells for each DNA content category is indicated. (g) Comparison of testicular morphology in wild type, $t p 53 \mathrm{~m} / \mathrm{m}, \mathrm{brca} 2 \mathrm{~m} / \mathrm{m}$, and $\mathrm{brca} 2 \mathrm{~m} / \mathrm{m}$; tp53 m/m zebrafish, Toluidine blue stain. Insets show type A (A, red) and type B (B, yellow) spermatogonia. Orange arrows indicate representative regions of stromal tissue and orange asterisks indicate examples of blood vessels within the stroma. (h) Comparison of the mean number of spermatogonia per 400X field (see Materials and Methods) in wild type, $t p 53 \mathrm{~m} / \mathrm{m}, \mathrm{brca} 2 \mathrm{~m} / \mathrm{m}$, and $\mathrm{brca} 2 \mathrm{~m} / \mathrm{m} ; \mathrm{tp} 53 \mathrm{~m} / \mathrm{m}$ zebrafish testes. The mean numbers of type A and type B spermatogonia are shown in the appropriate portion of each column. SG, spermatogonia; $*, \mathrm{p}=0.01-0.05 ; * *, \mathrm{p}=0.001-0.01 ; * * *, \mathrm{p}=0.0001-0.01 ; * * * *, \mathrm{p}<0.0001$. Error bars represent the range of the data. See Table S1 for specific p-values. 
chromosomal abnormalities [6, 7]. Additionally, disrupted interaction between Brca2 and the SAC mediator BubR1 resulted in both genomic instability and aneuploidy [8], and BRCA2 deficiency has been linked to defects in cytokineses $[9,10]$. BRCA2 may also participate in regulation of entry into mitosis after the G2 checkpoint [11, 12] and was found to be essential for protection of stalled replication forks [13]. These findings indicate that loss of functional BRCA2 severely disrupts both meiotic and mitotic cell cycles and has significant potential to destabilize genomic integrity.

The above studies predict that BRCA2-associated human cancers might exhibit a high prevalence of aneuploidy. However, comparison of BRCA2-associated and non-BRCA2associated human breast cancers has shown that BRCA2 mutation does not increase aneuploidy in human cancer [14-16]. Instead, diploid and aneuploid cancers occurred in roughly equal proportions in BRCA2 mutation carriers and noncarriers. Moreover, diploidy was identified as an independent negative prognostic indicator for BRCA2 mutation carriers that was linked to decreased survival time [16]. In contrast, diploidy was a positive prognostic indicator for noncarriers [16]. This observation is at odds with the fact that aneuploidy is generally considered to be a poor prognostic indicator for many human cancers [17-22]. These unexpected findings suggest an unusual and complex relationship between BRCA2 mutation, ploidy, and survival outcome.

In the current study, we used a zebrafish model to investigate the impact of BRCA2 mutation on meiotic and mitotic cell cycle outcomes and to assess the relationship between brca 2 mutation, ploidy, and survival in cancer-bearing zebrafish. The zebrafish brca2 ${ }^{Q 658 X}$ mutation is a nonsense mutation that is similar in location and type to pathologic BRCA2 mutations in humans [23]. The brca2-mutant zebrafish line is fully viable [23], unlike most Brca2-mutant mouse models (summarized by Evers B and Jonkers J) [24], and thus is useful for in vivo studies with adult animals. In human BRCA2-associated cancers, TP53 is frequently mutated, which is thought to be an early and essential step in survival of transformed cells [25-28]. Similarly, we previously showed that the zebrafish $t p 53^{M 214 K}$ mutation [29] exerts a collaborative effect on tumorigenesis in brca2-mutant zebrafish $[23,30]$. In the current study, we analyzed zebrafish siblings with and without brca2 mutation on a tp53-mutant background, enabling us to assess the specific impact of brca 2 mutation on ploidy outcome.

First, we determined the effect of brca2 and tp53 mutations on meiotic cell cycle progression in zebrafish testes by paired flow cytometry and histologic assessment. Second, we determined the influence of brca 2 and tp53 mutations on ploidy in zebrafish somatic cells and cancer cells and evaluated the contributions of other variables (sex, tumor location) to ploidy outcome. Finally, we identified the individual and combined impacts of brca2 genotype, sex, and tumor ploidy on survival outcome in cancer-bearing zebrafish.

\section{Materials and Methods}

2.1. Zebrafish Study Cohorts. Experiments were performed with adult wild type $(\mathrm{AB})$ zebrafish and adult zebrafish from the $b r c a 2^{h g 5}$ and $t p 53^{z d f 1}$ mutant zebrafish lines, corresponding to the $b r c a 2^{Q 658 X}$ and $t p 53^{M 214 K}$ mutations, respectively $[23,29]$. Mutant alleles for brca2 and tp53 are referred to as " $\mathrm{m}$ "; individual zebrafish within each genotypic group were siblings. For studies assessing zebrafish with or without brca $2^{Q 658 X}$ mutation on the $t p 53^{M 214 K}$ background, the compared study populations were composed of siblings. Thus, reference to $t p 53 \mathrm{~m} / \mathrm{m}$ zebrafish indicates siblings of brca2 $\mathrm{m} / \mathrm{m}$; tp53 $\mathrm{m} / \mathrm{m}$ zebrafish that do not carry the $b r c a 2^{\mathrm{Q} 658 \mathrm{X}}$ mutation. The study group used for analysis of tumor ploidy was composed of two related cohorts derived from two clutches in order to achieve a target of 50 individuals per genotype. As this target was not achieved with the first cohort, part of a second cohort was included in the study. All animal studies were approved by the Institutional Animal Care and Use Committee, North Carolina State University, Raleigh, $\mathrm{NC}$, and the methods were carried out in accordance with relevant guidelines and regulations.

2.2. Zebrafish Husbandry and Genotyping. Zebrafish used in this study were reared in a multirack recirculating containment system. From five to nine days of age, zebrafish larvae received live cultured Brachionus plicatilis (L-type rotifers), and, from ten to thirty days of age, zebrafish fry received commercially available, appropriately sized powder diets supplemented with live cultured Artemia sp. (brine shrimp). Juvenile and adult zebrafish received commercially available dry zebrafish diets supplemented with live cultured Artemia sp. Pathogen testing is performed on a biannual basis with the IDEXX Zebrafish Essential PCR Profile using zebrafish exposed to prefiltration water and swabs of detritus.

Zebrafish were monitored for clinical and gross evidence of tumor development and were collected in chronological order as tumors arose. Zebrafish were humanely euthanized with Tricaine methanesulfonate $(300 \mathrm{mg} / \mathrm{L})$ in system water buffered with Sodium Bicarbonate to a $\mathrm{pH}$ of $\sim 7.0$. Live adult zebrafish were genotyped for the $\operatorname{brca} 2^{\mathrm{Q} 658 \mathrm{X}}$ mutation by sequencing over the mutation site as described previously [31]. Zebrafish on the $t p 53^{M 214 K}$ background were maintained as a homozygous mutant line.

2.3. Tissue Collection and Histologic Analysis. Normal and tumor tissues were identified and collected by dissection using a stereomicroscope. For DNA content analysis, tissue samples were prepared as described below. For histologic analysis of tumor-bearing zebrafish, a sample of tumor tissue and the coelomic viscera were collected and fixed in $4 \%$ Paraformaldehyde. Fixed tissues were routinely processed for decalcification as needed, paraffin embedding, and preparation of hematoxylin- and eosin-stained sections. For histologic analysis of zebrafish testes, fixed tissues were embedded in glycol methacrylate, sectioned at $2.5 \mu \mathrm{m}$ thickness, and stained with Toluidine blue stain $(0.01 \mathrm{~g} / \mathrm{ml}$ Toluidine blue and $0.01 \mathrm{~g} / \mathrm{ml}$ sodium tetraborate in distilled water).

Histologic sections were analyzed with an Olympus BX43 microscope and imaged with a DP26 digital camera and cellSens entry microscope imaging software, version 1.5. Histologic images were minimally and globally 
processed for exposure, contrast, and/or color balance with the GNU Image Manipulation Program, version 2.8.6 (http://www.gimp.org/).

For quantification of spermatogonia in zebrafish testes, three representative images were captured at $400 \mathrm{X}$ from the testes of five zebrafish from each genotypic group for a total of 15 histologic sections per genotypic group. For one brca2 $\mathrm{m} / \mathrm{m}$; tp53 $\mathrm{m} / \mathrm{m}$ zebrafish, only two representative images were quantified due to insufficient tissue for capturing three high-quality images; thus, a total of 14 histologic sections were evaluated for this genotypic group. Spermatogonia were manually counted in each digital image using the ImageJ Fiji Cell Counter tool [32]. Spermatogonia were identified by histologic characteristics as previously described [33], and type A and type B spermatogonia were counted separately.

2.4. DNA Content Analysis. For preparation of dissociated zebrafish testes, both testes from each zebrafish were collected, minced, and incubated in $500 \mathrm{U} / \mathrm{ml}$ Collagenase (Collagenase type I in 1X Hank's Balanced Salt Solution in $\mathrm{L} 15$ medium) at $28^{\circ} \mathrm{C}$ for 2 hours with gentle pipetting every 20 minutes. For preparation of dissociated nonneoplastic somatic cells and cancer cells, matched normal and tumor tissues samples from each individual zebrafish were collected and dissociated as described above. Dissociated cells were washed with 1X phosphate-buffered saline (PBS), filtered with a $35 \mu \mathrm{m}$ filter and fixed with ice-cold $70 \%$ ethanol. Cell suspensions were maintained at $-20^{\circ} \mathrm{C}$ for a minimum of 24 hours. After fixation, cell suspensions were washed with $1 X$ PBS and stained with Propidium Iodide staining solution containing RNase (Cellometer PI Cell Cycle Kit, CSK-0112, Nexcelom, Lawrence, MA).

Cell suspensions were analyzed for DNA content with a Beckman Coulter CytoFLEX flow cytometer. The CytoFLEX was maintained and calibrated daily according to the manufacturer's recommendations. Up to 10,000 events were recorded per sample at a flow rate of $10-30 \mu \mathrm{l} / \mathrm{min}$ (up to 300 events/second). Matched normal and cancer specimens from individual zebrafish were analyzed during the same experiment.

Flow cytometry data were analyzed with DeNovo FCS Express 6 Flow Research Edition. For DNA content analysis of testes, the gating strategy was based on the method described by Rotgers et al. [34]. Cell suspensions from zebrafish testes were gated on forward scatter-A (FSC-A) versus FSC-H, followed by gating on Propidium Iodide-A (PI-A) versus FSC-A (Figure S1A). DNA histograms were generated using PI-H, and haploid (1C), diploid (2C), Sphase, and tetraploid (4C) populations were identified as previously described [34]. Percentages of each cell populations identified by DNA content were acquired by defining marker gates for each population (Figure S1A).

For DNA content analysis of cell suspensions from nonneoplastic somatic tissues and cancer tissues, cells were gated on PI-H versus PI-A. DNA histograms were generated using PI-H with the FCS Express 6 Multicycle AV Professional Version (Figure S1B). Cell cycles were modeled with the SL S0 model (sliced nuclei background modeling with zero order $S$ phase). At least 1,000 events were analyzed to generate the cell cycle for all but one nonneoplastic somatic tissue specimen, for which 632 events were analyzed.

2.5. Criteria for Exclusion of Samples. DNA content analysis was attempted on testes from age-matched zebrafish in order to meet a target of at least five individuals per genotypic group. Individual results were excluded from the study under the following criteria: insufficient cell number to generate cell cycle profile.

DNA content analysis was attempted on normal and tumor specimens from zebrafish in chronological order, as cancers arose, in order to meet a target of 50 individuals per genotype. Individuals were excluded from the study under the following criteria: (1) found dead; (2) no grossly identifiable tumor tissue; (3) insufficient cell number to generate cell cycle profile; (4) coefficient of variance (CV) of the sample from nonneoplastic somatic tissue $>6.0$; (5) inability to define the diploid population in the nonneoplastic somatic tissue specimen.

2.6. Calculation of Tumor Ploidy. Tumor ploidy was defined by calculating the DNA index (Table S1) [35]. For tumor samples that contained an internal diploid population with a $C V \leq 6.0$, the DNA index was calculated using the G0/G1 peak fluorescence intensity value of the internal diploid population. The internal diploid population was confirmed to be diploid by comparing the G0/G1 peak fluorescence intensity value of this population to the G0/G1 peak fluorescence intensity value of the matched nonneoplastic somatic tissue specimen. For tumor samples that did not contain an internal diploid population or contained an internal diploid population with a $\mathrm{CV}>6.0$, the DNA index was calculated using the G0/G1 peak fluorescence intensity value of the matched nonneoplastic somatic tissue specimen. Tumors that exhibited multiple peaks were defined as complex aneuploid and a DNA index was not calculated.

For five zebrafish cancers $(4 \mathrm{brca} 2 \mathrm{~m} / \mathrm{m} ; \mathrm{tp} 53 \mathrm{~m} / \mathrm{m}$ and 1 tp53 $\mathrm{m} / \mathrm{m})$, an aneuploid population was inconsistently modeled or constituted $\leq 20 \%$ of the total population. For eight zebrafish cancers $(5 \mathrm{brca} 2 \mathrm{~m} / \mathrm{m}$; tp53 $\mathrm{m} / \mathrm{m}$ and 3 tp53 $\mathrm{m} / \mathrm{m}$ ), a subpopulation of cells was inconsistently modeled as either an aneuploid population or as the G2/M population. For these thirteen cancers, the G0/G1 peak fluorescence intensity value of the predominant diploid population was used to calculate DNA index.

2.7. Statistical Analyses. Statistical analyses were performed using JMP Pro 13.2.1 (SAS Institute Inc.). Statistical significance was set at an alpha value of $\mathrm{p} \leq 0.05$. Comparisons of zebrafish testes were performed between zebrafish exhibiting complete spermatogenesis (wild type and $t p 53 \mathrm{~m} / \mathrm{m}$ ) and incomplete spermatogenesis (brca $2 \mathrm{~m} / \mathrm{m}$ and brca $2 \mathrm{~m} / \mathrm{m}$; tp53 $\mathrm{m} / \mathrm{m}$ ). The percentages of cells by DNA content category in zebrafish testes and nonneoplastic somatic tissues were compared by t-test corrected for unequal variances. The four samples from nonneoplastic tissues that exhibited a small aneuploid peak, described above, were excluded from comparison of the percent gated cells in G0/G1, S, and $\mathrm{G} 2 / \mathrm{M}$ phases. The numbers of spermatogonia in zebrafish 
testes were compared by unpaired t-test assuming unequal variances. Comparison of G1 peak PI fluorescence intensity values were tested for normality by fitting a normal distribution and analyzing goodness-of-fit (Shapiro-Wilk W test). Samples from Experiment Seven that exhibited anomalously high G0/G1 peak PI fluorescence intensity values, described below, were excluded from this analysis ( 5 samples from brca2 $\mathrm{m} / \mathrm{m}$; tp53 $\mathrm{m} / \mathrm{m}$ zebrafish, 1 sample from tp53 $\mathrm{m} / \mathrm{m}$ zebrafish). The Chi-square test was used to test for associations in pairwise comparisons of genotype, tumor location, ploidy outcome, and sex. The median survival times were obtained using the Kaplan-Meier test and differences in survival curves were assessed by the log-rank test and Cox's Proportional Hazard Model. Cox's Proportional Hazard Model was used to determine contribution to survival by the purported risk variables (i.e., brca2 mutation status, sex, and tumor ploidy).

\section{Results}

3.1. Combined brca2 and tp53 Mutations Induce Meiotic Arrest and Spermatogonial Expansion in Zebrafish. We previously showed that zebrafish with homozygous brca2 $2^{\text {Q658X }}$ mutation $($ brca $2 \mathrm{~m} / \mathrm{m}$ ) develop exclusively as males and exhibit incomplete spermatogenesis with extensive spermatocyte apoptosis, reflecting a conserved role for BRCA2 in germ cell development [23]. In comparison, homozygous tp53 $3^{M 214 K}$ mutation $($ tp53 $\mathrm{m} / \mathrm{m})$ was not reported to impact sex ratios or fertility in zebrafish [29]. In the following studies, comparisons were performed between testes with complete spermatogenesis (wild type and tp53 $\mathrm{m} / \mathrm{m}$ ) and between testes with incomplete spermatogenesis $($ brca $2 \mathrm{~m} / \mathrm{m}$ and $\operatorname{brca} 2 \mathrm{~m} / \mathrm{m} ; \mathrm{tp} 53 \mathrm{~m} / \mathrm{m}$ ).

Similar to $b r c a 2 \mathrm{~m} / \mathrm{m}$ zebrafish, brca2 $\mathrm{m} / \mathrm{m}$; tp53 $\mathrm{m} / \mathrm{m}$ males are sterile and exhibit incomplete spermatogenesis, with only spermatogonia and primary spermatocytes present in testes (Figure S2A). In comparison, tp53 $\mathrm{m} / \mathrm{m}$ male zebrafish are fertile and exhibit complete spermatogenesis, with no histologic abnormalities observed in testes (Figure S2B). To further investigate this phenotype, we analyzed dissociated testes from age-matched wild type $(\mathrm{n}=5), t p 53$ $m / m(\mathrm{n}=7), \operatorname{brca} 2 \mathrm{~m} / \mathrm{m}(\mathrm{n}=9)$, and $\mathrm{brca} 2 \mathrm{~m} / \mathrm{m}$; tp53 m/m $(\mathrm{n}=$ 5) male zebrafish by flow cytometry (Figure 1 and Figure S1A). Testes from wild type and $t p 53 \mathrm{~m} / \mathrm{m}$ zebrafish had similar cell cycle profiles and exhibited a predominance of cells with 1C DNA content, representing mature spermatozoa (Figures 1(b), 1(c), and 1(f)). The proportions of cells in each DNA content category (1C, 2C, S, and $4 \mathrm{C}$ ) were not significantly different in testes from wild type and tp53 $\mathrm{m} / \mathrm{m}$ zebrafish, with the exception of the $2 \mathrm{C}$ population $(\mathrm{p}=0.0405$, unpaired $\mathrm{t}$ test; Figure 1(f) and Table S1).

In contrast, testes from $b r c a 2 \mathrm{~m} / \mathrm{m}$ and $b r c a 2 \mathrm{~m} / \mathrm{m}$; tp53 $\mathrm{m} / \mathrm{m}$ zebrafish did not contain an appreciable cell population with 1C DNA content ( $<2 \%$ of gated cells), indicating arrested spermatogenesis in males from these genotypic groups. Seven of 9 testes from $\mathrm{brca} 2 \mathrm{~m} / \mathrm{m}$ zebrafish exhibited a predominance of cells with 4C DNA content (Figures $1(\mathrm{~d})$ and $1(\mathrm{f})$ ), while all testes from $b r c a 2 \mathrm{~m} / \mathrm{m} ; \mathrm{tp} 53 \mathrm{~m} / \mathrm{m}$ zebrafish exhibited a predominance of cells with $2 \mathrm{C}$ DNA content
(Figures 1(e) and 1(f)). Testes from brca2 $\mathrm{m} / \mathrm{m}$ zebrafish additionally exhibited an increased proportion of cells in $\mathrm{S}$ phase. The proportions of cells in each DNA content category (2C, S, and 4C) were significantly different in testes from brca $2 \mathrm{~m} / \mathrm{m}$ and brca $2 \mathrm{~m} / \mathrm{m}$; tp53 $\mathrm{m} / \mathrm{m}$ zebrafish (Figure 1(f) and Table S1).

We next sought to determine a cause for the difference in the proportions of cells with $2 \mathrm{C}$ versus $4 \mathrm{C}$ DNA content in testes from brca $2 \mathrm{~m} / \mathrm{m}$ versus brca $2 \mathrm{~m} / \mathrm{m}$; tp53 $\mathrm{m} / \mathrm{m}$ zebrafish. The $2 \mathrm{C}$ population identified by flow cytometry in dissociated testes includes spermatogonia, secondary spermatocytes, and somatic cells (stromal component). However, secondary spermatocytes are rarely observed in zebrafish testes due to rapid entry into meiosis II [33]. We performed quantitative histologic analysis on thin sections of testes to determine the prevalence of spermatogonia. We analyzed testes from age-matched wild type $(\mathrm{n}=5)$, tp53 $\mathrm{m} / \mathrm{m}$ (n = 5), brca2 $\mathrm{m} / \mathrm{m}(\mathrm{n}=5)$, and brca $2 \mathrm{~m} / \mathrm{m}$; tp53 $\mathrm{m} / \mathrm{m}(\mathrm{n}=$ 5) male zebrafish (Figure $1(\mathrm{~g})$ ). Because testes from brca2 $\mathrm{m} / \mathrm{m}$ and $\mathrm{brca} 2 \mathrm{~m} / \mathrm{m}$;tp53 $\mathrm{m} / \mathrm{m}$ zebrafish do not contain spermatozoa, testicular tubules are generally smaller and closer together than in testes from wild type and tp53 m/m zebrafish (Figure $1(\mathrm{~g})$ ). We therefore compared the numbers of spermatogonia between genotypic groups with complete spermatogenesis (wild type and tp53 $\mathrm{m} / \mathrm{m}$ ) and between genotypic groups with arrested spermatogenesis $($ brca $2 \mathrm{~m} / \mathrm{m}$ and brca $2 \mathrm{~m} / \mathrm{m} ; \mathrm{tp} 53 \mathrm{~m} / \mathrm{m}$ ) (Figure $1(\mathrm{~h})$ and Table S1).

In mammals [36] and zebrafish [33], spermatogonia can be identified as type A or type B based on nuclear morphology, with type A representing a less differentiated population than type B. $t p 53 \mathrm{~m} / \mathrm{m}$ testes exhibited a significantly increased number of spermatogonia compared to wild type testes that was attributable to expansion of the type A spermatogonial population $(\mathrm{p}<0.0001$, unpaired $\mathrm{t}$ test; Figure $1(\mathrm{~h})$ and Table S1). brca $2 \mathrm{~m} / \mathrm{m} ; \mathrm{tp} 53 \mathrm{~m} / \mathrm{m}$ testes exhibited a significantly increased number of spermatogonia compared to brca $2 \mathrm{~m} / \mathrm{m}$ testes. This increase was largely attributable to expansion of the type A spermatogonial population, although the type $B$ spermatogonial populations were also significantly increased (type A, p $<0.0001$, unpaired t-test; type B, p $=0.0061$, unpaired t-test; Figure $1(\mathrm{~h})$ and Table S1). In brca $2 \mathrm{~m} / \mathrm{m}$; tp53 $\mathrm{m} / \mathrm{m}$ testes, we observed occasional giant spermatogonia (Figure S2C) and germ cells that were morphologically consistent with perinucleolar oocytes (Figure S2D). We did not observe these cell types in any other genotypic group. The stromal component of the testes (containing various types of somatic cells) appeared similar between zebrafish of different genotypes (Figure $1(\mathrm{~g})$ ).

3.2. brca2 Mutation Does Not Alter Ploidy or Cell Cycle Progression in Nonneoplastic Zebrafish Somatic Cells. In preparation for analyzing ploidy in zebrafish cancers, we collected matched nonneoplastic somatic tissue from each cancerbearing zebrafish. This enabled us to define the diploid population for calculation of tumor ploidy for each cancer specimen and also allowed us to assess the impact of brca2 mutation on DNA content and cell cycle progression in nonneoplastic somatic cells. We analyzed somatic tissues from $49 \mathrm{brca} 2 \mathrm{~m} / \mathrm{m} ; \mathrm{tp} 53 \mathrm{~m} / \mathrm{m}$ zebrafish and 50 tp53 $\mathrm{m} / \mathrm{m}$ 
TABLE 1: Characteristics of the study population used for analysis of somatic and cancer cells.

\begin{tabular}{|c|c|c|}
\hline & brca $2 \mathrm{~m} / \mathrm{m} ; \mathrm{tp} 53 \mathrm{~m} / \mathrm{m}$ & $t p 53 \mathrm{~m} ; \mathrm{m}$ \\
\hline Total zebrafish & 49 & 50 \\
\hline Males & $22(45 \%)$ & $25(50 \%)$ \\
\hline Females & $26(53 \%)$ & $25(50 \%)$ \\
\hline Sex not determined & $1(2 \%)$ & $0(0 \%)$ \\
\hline Total tumors ${ }^{\mathrm{a}}$ & 52 & 51 \\
\hline \multicolumn{3}{|l|}{ Age at tumor diagnosis (mo) } \\
\hline Median age (total population) & 8.2 & 10.8 \\
\hline Range (total population) & $6.0-13.1$ & $7.1-13.9$ \\
\hline Median age (males) & 9.0 & 11.3 \\
\hline Range (males) & $6.2-13.1$ & $7.1-13.9$ \\
\hline Median age (females) & 7.4 & 9.7 \\
\hline Range (females) & $6.0-10.1$ & $7.1-13.6$ \\
\hline \multicolumn{3}{|l|}{ Tumor location } \\
\hline Coelom & $30(58 \%)$ & $40(78 \%)$ \\
\hline Ocular region & $19(37 \%)$ & $9(18 \%)$ \\
\hline Other & $3(6 \%)$ & $2(4 \%)$ \\
\hline
\end{tabular}

${ }^{a}$ Two anatomically distinct tumors were independently analyzed for three $b r c a 2 \mathrm{~m} / \mathrm{m} ; \mathrm{tp} 53 \mathrm{~m} / \mathrm{m}$ zebrafish and one tp53 $\mathrm{m} / \mathrm{m}$ zebrafish.

zebrafish for DNA content (Table 1 and Figure S2B). 46 tissue samples from $b r c a 2 \mathrm{~m} / \mathrm{m}$; $553 \mathrm{~m} / \mathrm{m}$ zebrafish and 49 samples from tp53 $\mathrm{m} / \mathrm{m}$ zebrafish exhibited a single diploid cell cycle. Four nonneoplastic somatic tissues exhibited a small aneuploid peak (ranging from 8.8 to $16.0 \%$ of gated cells) in addition to a predominant diploid cell cycle. These tissues were derived from three brca $2 \mathrm{~m} / \mathrm{m}$; tp53 $\mathrm{m} / \mathrm{m}$ zebrafish and one tp53 $\mathrm{m} / \mathrm{m}$ zebrafish.

Comparison of the individual diploid G0/G1 peak propidium iodide (PI) fluorescence intensity values for nonneoplastic somatic cells demonstrated tight clustering of individual values within and between each independent flow cytometry analysis, designated as Experiments 1-23 (Figure 2(a), upper panel). Samples analyzed in one flow cytometry analysis (Experiment 7) showed anomalously high G0/G1 peak PI fluorescence intensity values compared to other samples but were highly similar to one another. Overall, the distributions of G0/G1 peak PI fluorescence intensity values were similar for nonneoplastic somatic cells derived from $\mathrm{brca} 2 \mathrm{~m} / \mathrm{m}$; $t$ 553 $\mathrm{m} / \mathrm{m}$ and tp53 $\mathrm{m} / \mathrm{m}$ zebrafish, both within and between each independent flow cytometry analysis. In contrast, the distributions of G0/G1 peak PI fluorescence intensity values for cancer cells derived from $b r c a 2 \mathrm{~m} / \mathrm{m} ; \mathrm{tp} 53 \mathrm{~m} / \mathrm{m}$ and $t p 53$ $\mathrm{m} / \mathrm{m}$ zebrafish were highly variable (Figure 2(a), lower panel).

We analyzed the individual G0/G1 peak PI fluorescence intensity values for nonneoplastic somatic cells derived from brca $2 \mathrm{~m} / \mathrm{m}$; tp53 $\mathrm{m} / \mathrm{m}$ and tp53 $\mathrm{m} / \mathrm{m}$ zebrafish for a normal distribution (Figure 2(b)). For both genotypic groups, the G0/G1 peak PI fluorescence intensity values followed a normal distribution, and the median G0/G1 peak PI fluorescence intensity values were similar (Figure 2(b)).

We assessed cell cycle progression in nonneoplastic somatic tissues based on DNA content distribution by comparing the percent gated cells in G0/G1, S, and G2/M phases (Figure S3). The mean percentages of cells in G0/G1, S, and
G2/M phases were similar for brca $2 \mathrm{~m} / \mathrm{m}$; tp53 $\mathrm{m} / \mathrm{m}$ and tp53 $\mathrm{m} / \mathrm{m}$ zebrafish (Figure S3A).

3.3. Tumor Ploidy Is Not Significantly Different in brca2Associated and Non-brca2-Associated Zebrafish Cancers. We performed DNA content analysis on cancers derived from $49 \mathrm{brca} 2 \mathrm{~m} / \mathrm{m}$; tp53 $\mathrm{m} / \mathrm{m}$ zebrafish and 50 tp53 $\mathrm{m} / \mathrm{m}$ zebrafish siblings (Figures 3(a)-3(d) and Table 1). Cancer-bearing zebrafish in these studies were siblings that were distinguished by presence or absence of the brca2 mutation. Therefore, cancers arising in brca $2 \mathrm{~m} / \mathrm{m} ; \mathrm{tp} 53 \mathrm{~m} / \mathrm{m}$ zebrafish are referred to as brca2-associated cancers and cancers arising in $t p 53 \mathrm{~m} / \mathrm{m}$ zebrafish are referred to as non- $b r c a 2$-associated cancers. Cancers were predominantly soft tissue sarcomas that showed variable histologic differentiation toward malignant peripheral nerve sheath tumor (MPNST), as we have previously described in zebrafish of these genotypes [31]. We have previously shown that brca2 genotype was not correlated to degree of histologic differentiation (poorly differentiated sarcoma versus well-differentiated MPNST) [31]. In comparison, we have previously shown that brca2 $\mathrm{m} / \mathrm{m}$ zebrafish without concurrent tp53 mutation exhibit a relative increase in the incidence of benign testicular tumors $[23,30]$.

For each cancer-bearing zebrafish, matched nonneoplastic somatic tissues were simultaneously analyzed as described above. In three brca $2 \mathrm{~m} / \mathrm{m} ;$ tp53 $\mathrm{m} / \mathrm{m}$ zebrafish and one tp53 $\mathrm{m} / \mathrm{m}$ zebrafish, there were two anatomically distinct tumors (e.g., both an ocular and a coelomic tumor in one individual) that were collected and analyzed independently. In total, 52 cancers from brca $2 \mathrm{~m} / \mathrm{m}$; tp $53 \mathrm{~m} / \mathrm{m}$ zebrafish and 51 cancers from $t p 53 \mathrm{~m} / \mathrm{m}$ zebrafish were analyzed. Ploidy was determined by calculating the DNA index based on G0/G1 peak PI fluorescence intensity values (Table S2 and Methods). 


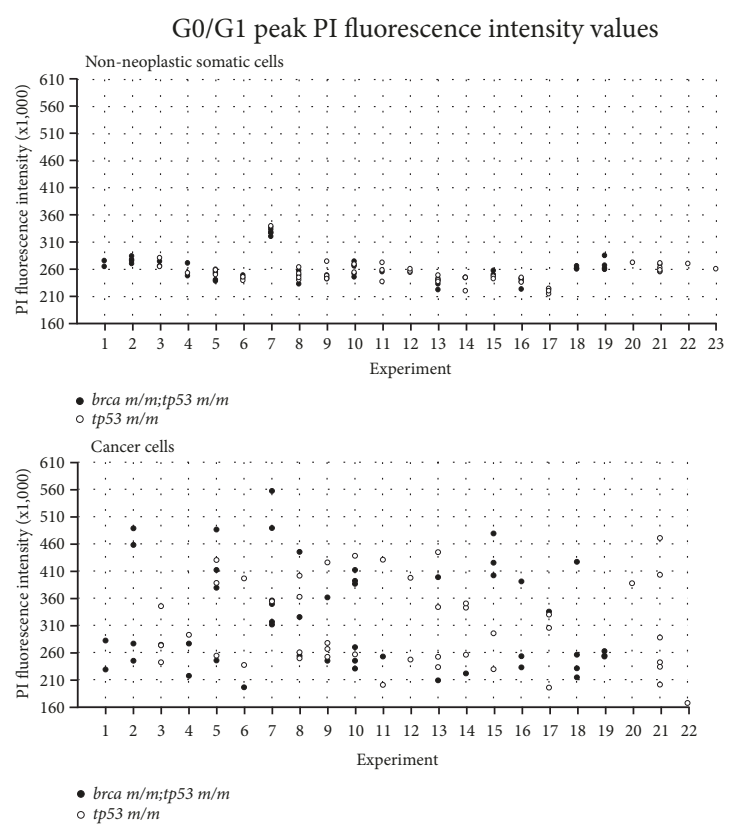

(a)

- $b r c a m / m ; t p 53 \mathrm{~m} / \mathrm{m}$
Distribution of G0/G1 peak PI fluorescence intensity values
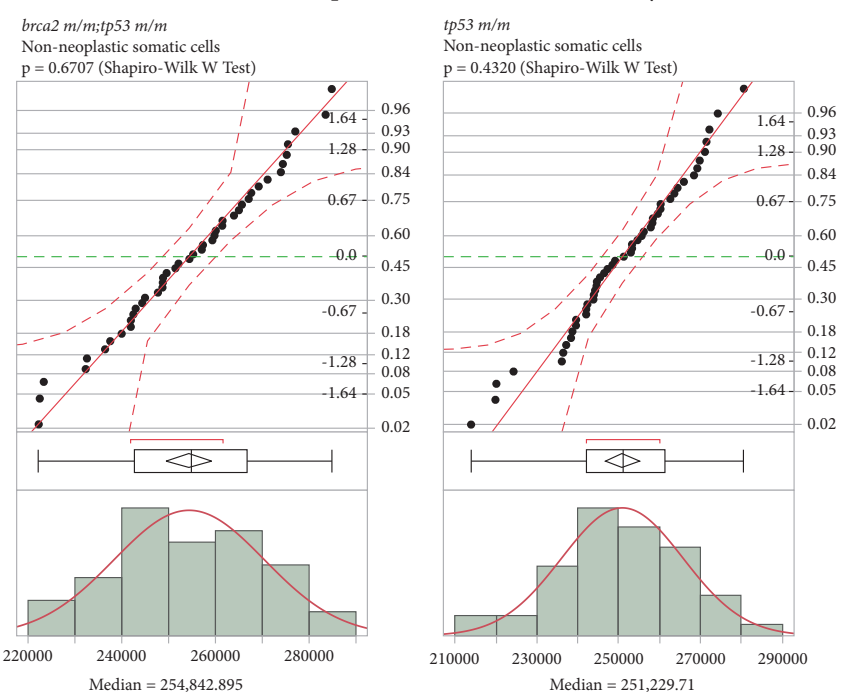

(b)

FIGURE 2: brca2 mutation does not alter DNA content of nonneoplastic somatic zebrafish tissue. (a) Comparison of G0/G1 peak propidium iodide (PI) fluorescence intensity values for nonneoplastic somatic cells (upper panel) and cancer cells (lower panel) derived from 49 brca2 $\mathrm{m} / \mathrm{m} ; \mathrm{tp} 53 \mathrm{~m} / \mathrm{m}$ (black circles) and 50 tp53 m/m (white circles) cancer-bearing zebrafish. Each circle indicates the G0/G1 peak value for a single sample. Two matched samples (nonneoplastic somatic cells and cancer cells) were analyzed from each individual zebrafish. For every individual zebrafish, the matched nonneoplastic somatic cell sample and cancer cell sample were analyzed in the same experiment. The experiment number indicated on the $x$-axis refers to each independent cell cycle analysis. In experiment 23 , the cancer cell sample was excluded (see Materials and Methods), and the G0/G1 peak is only reported for the matched nonneoplastic somatic cell sample. (b) Normal distribution of G0/G1 peak PI fluorescence intensity values for nonneoplastic somatic cells derived from $\mathrm{brca} 2 \mathrm{~m} / \mathrm{m} ; \mathrm{tp} 53 \mathrm{~m} / \mathrm{m}$ and $t p 53$ $\mathrm{m} / \mathrm{m}$ zebrafish. In the normal quantile plot, filled black circles represent individual data points and dashed red lines indicate the Lilliefores confidence bounds. In the outlier box plot, the vertical line represents the median sample value; the diamond contains the mean and upper and lower $95 \%$ of the mean; the box ends represent the $25^{\text {th }}$ and $75^{\text {th }}$ quantiles; the whiskers extend to the outermost data points; and the red bracket indicates the shortest half (most dense $50 \%$ of observations). In the histogram, vertical bars represent G0/G1 peak intensity values by bin and the overlying red curve fits a smooth curve using nonparametric density estimation.

Based on DNA index, zebrafish cancers were classified as diploid or aneuploid, and aneuploid tumors were further categorized by type of aneuploidy (Table S2 and Table 2). brca2 genotype influenced the relative proportions of cancers in diploid and aneuploid categories (Figure 3(b) and Table 2). For brca2-associated cancers, approximately equal proportions of cancers were diploid versus aneuploid (48\% and $52 \%$, respectively). In contrast, for non-brca2-associated cancers the proportion of diploid cancers was almost half the proportion of aneuploid cancers (35\% and 65\%, respectively). However, the association between brca2 genotype and ploidy outcome (diploid versus aneuploid) was not statistically significant $(\mathrm{p}=0.1877$, Chi-square test). For both brca2associated and non-brca2-associated cancers, hyperdiploid aneuploidy was the most common nondiploid categorization (Table 2).

Four zebrafish (three brca2 $\mathrm{m} / \mathrm{m}$; tp53 $\mathrm{m} / \mathrm{m}$ and one tp53 $\mathrm{m} / \mathrm{m}$ zebrafish) developed two cancers in anatomically distinct locations that were analyzed independently (Figure S4). In three of four individuals, the two cancers did not exhibit the same ploidy (aneuploid versus diploid). In one of four individuals, the two cancers exhibited the same ploidy (both aneuploid).

We assessed cell cycle progression in zebrafish cancers based on DNA content distribution (Figure S3). The mean percentage of cells in G0/G1, S, and G2/M phases were similar for diploid cancers from $b r c a 2 \mathrm{~m} / \mathrm{m}$; tp53 $\mathrm{m} / \mathrm{m}$ and tp53 $\mathrm{m} / \mathrm{m}$ zebrafish (Figure S3B) and for aneuploid cancers from brca $2 \mathrm{~m} / \mathrm{m} ; \mathrm{tp} 53 \mathrm{~m} / \mathrm{m}$ and tp53 $\mathrm{m} / \mathrm{m}$ zebrafish (Figure $\mathrm{S} 3 \mathrm{C})$.

3.4. Sex, But Not Tumor Location, Significantly Influences Ploidy Outcome in Zebrafish Cancers. We have previously shown that the coelom and ocular region are the most common sites for cancer development in zebrafish with brca 2 and tp53 mutations [23, 31], similar to the $t p 53^{M 214 K}$ line [29]. In the current study populations, the majority of analyzed cancer specimens arose in coelomic or ocular locations, with a small number arising in other locations (Table 1). We observed approximately twice as many ocular cancers in brca $2 \mathrm{~m} / \mathrm{m}$; tp53 $\mathrm{m} / \mathrm{m}$ zebrafish as occurred in tp53 $\mathrm{m} / \mathrm{m}$ zebrafish (Table 1). The association between brca2 genotype 

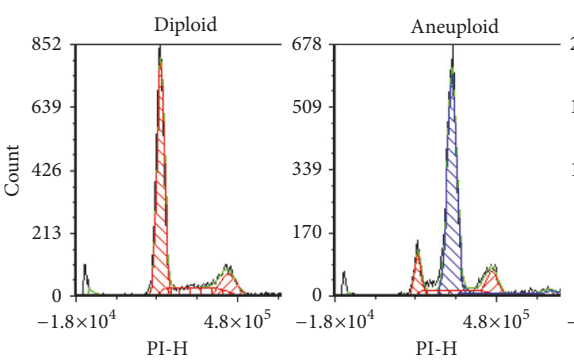

(a)

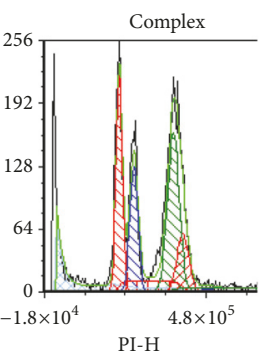

I-H

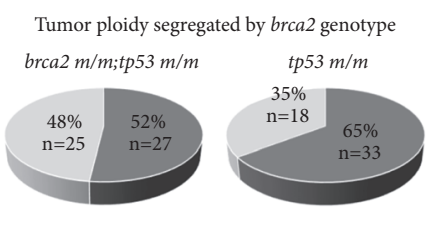

$\square$ Diploid

$\square$ Aneuploid

(b)

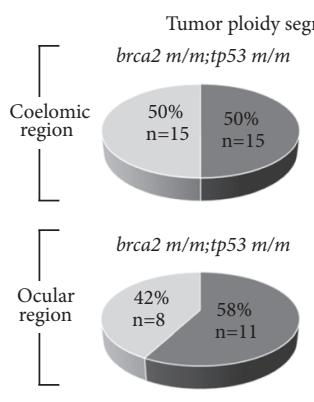

$\square$ Diploid

$\square$ Aneuploid

(c)

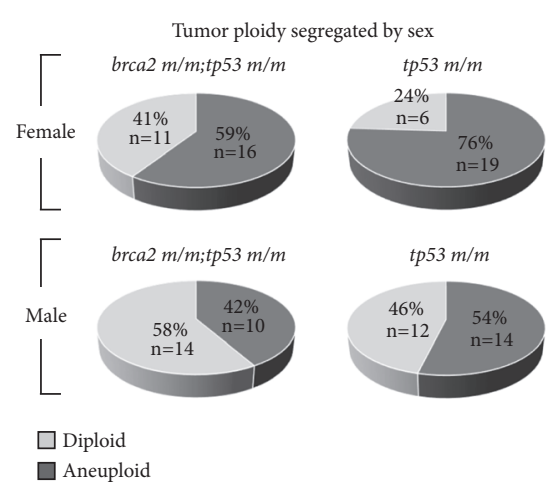

(d)

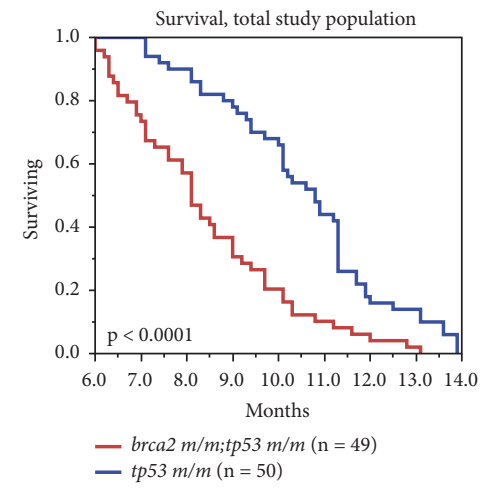

(e)

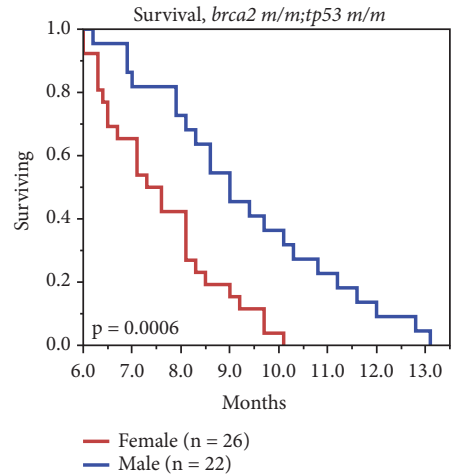

(f)

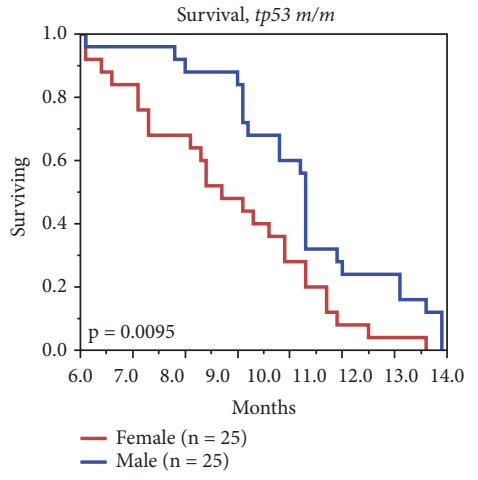

(g)

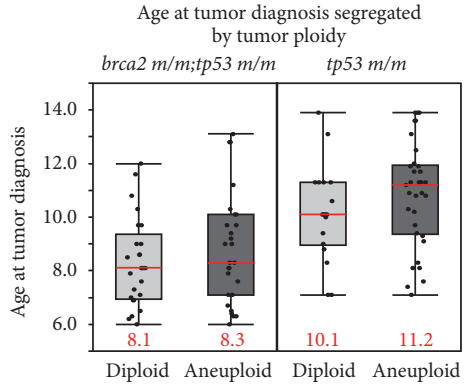

(h)

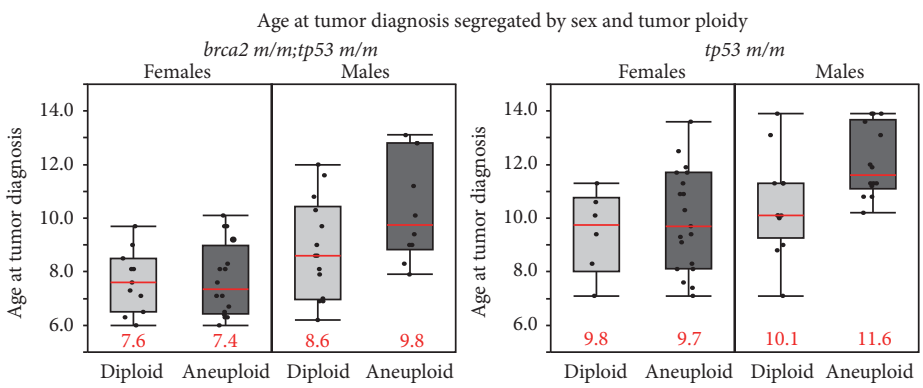

(i)

FIGURE 3: brca2 mutation status and sex alter ploidy outcomes in zebrafish cancers. (a) Representative propidium iodide (PI) fluorescence histograms from cancers that are diploid or aneuploid or exhibit complex aneuploidy. Software-identified diploid populations are depicted in red; aneuploid populations are depicted in blue and green. ((b)-(d)) Ploidy outcomes segregated by brca2 genotype alone (b) and in combination with tumor location (c) or sex (d). ((e)-(g)) Kaplan-Meier survival curves for the total study population (e), the $b r c a 2 m / m ; t p 53$ $\mathrm{m} / \mathrm{m}$ cohort (f), and the tp53 $\mathrm{m} / \mathrm{m}$ cohort (g). (h) Distribution of ages at tumor diagnosis segregated by brca2 genotype and tumor ploidy. Median ages at tumor diagnosis are indicated by a red bar and are shown in red text. (i) Distribution of ages at tumor diagnosis segregated by brca2 genotype, sex, and tumor ploidy. Median ages at tumor diagnosis are indicated by a red bar and shown in red text. 
TABLE 2: Impact of brca2 genotype and sex ${ }^{\mathrm{a}}$ on the relative proportions of zebrafish cancers in diploid and aneuploid categories.

\begin{tabular}{lcc}
\hline Ploidy category & brca2 m/m;tp53 m/m & $t p 53 m ; m$ \\
\hline Diploid & $25(48 \%)$ & $18(35 \%)$ \\
\hline Aneuploid & $27(52 \%)$ & $33(65 \%)$ \\
\hline Hypodiploid aneuploid & 4 & 3 \\
\hline Hyperdiploid aneuploid & 19 & 23 \\
\hline Tetraploid aneuploid & 1 & 0 \\
\hline Complex & 3 & 7 \\
\hline Ploidy category ${ }^{\text {b }}$ & Females & Males \\
\hline Diploid & $17(33 \%)$ & $26(52 \%)$ \\
\hline Aneuploid & $35(67 \%)$ & $24(48 \%)$ \\
\hline Hypodiploid aneuploid & 4 & 3 \\
\hline Hyperdiploid aneuploid & 25 & 16 \\
\hline Tetraploid aneuploid & 1 & 0 \\
\hline Complex & 5 & 5 \\
\hline
\end{tabular}

${ }^{\mathrm{a}}$ The sex for one zebrafish was not determined and is not included in the comparison of ploidy in females versus males.

${ }^{\mathrm{b}}$ Categorization of ploidy outcomes by sex includes all cancer-bearing zebrafish without segregation by brca2 genotype. Ploidy outcomes segregated by both sex and genotype are presented in Figure 3(d).

and tumor location (coelom or ocular region) was statistically significant $(\mathrm{p}=0.0241$, Chi-square test $)$.

To determine if the site of tumor origin influenced ploidy outcome, we assessed the numbers of cancers in each ploidy category arising in coelomic versus ocular locations (Figure 3(c)). Because brca2 genotype significantly influenced tumor location, ploidy outcomes were assessed only within genotypic groups. For cancers arising in the coelomic region, brca2-associated cancers were classified as diploid or aneuploid in equal proportions ( $\mathrm{n}=15,50 \%$, for both categories). Non-brca2-associated coelomic cancers were predominantly classified as aneuploid $(\mathrm{n}=27,68 \%)$. These outcomes were similar to the ploidy outcomes for each genotypic group (Figure 3(b)). For cancers arising in the ocular region, both brca2-associated and non-brca2-associated cancers were classified as diploid or aneuploid in relatively similar proportions. There was no statistically significant association between location and ploidy outcome (diploid versus aneuploid) for either genotypic group ( $\mathrm{brca} 2 \mathrm{~m} / \mathrm{m} ; \mathrm{tp} 53$ $m / m, p=0.5890 ; t p 53 \mathrm{~m} / \mathrm{m}, \mathrm{p}=0.0 .1545$; Chi-square test).

To determine the effect of sex on ploidy outcome in zebrafish cancers, we assessed the numbers of cancers in each ploidy category for male and female zebrafish (Figure 3(d) and Table 2). The numbers of males and females were similar in each genotypic group (Table 1) and there was no statistically significant association between brca2 genotype and sex $(\mathrm{p}=0.6920$, Chi-square test). Therefore, ploidy outcomes were assessed within genotypic groups and within the entire study population. In both $\mathrm{brca} 2 \mathrm{~m} / \mathrm{m} ; \mathrm{tp} 53 \mathrm{~m} / \mathrm{m}$ and $t p 53 \mathrm{~m} / \mathrm{m}$ cohorts, the proportions of aneuploid cancers were higher in females than in males of the same genotype (Figure $3(\mathrm{~d})$ ). In the brca2 $\mathrm{m} / \mathrm{m} ; \mathrm{tp} 53 \mathrm{~m} / \mathrm{m}$ cohort, $59 \%$ of cancers in females were aneuploid $(\mathrm{n}=16)$ versus $42 \%$ in males $(\mathrm{n}=10)$. In the tp53 $\mathrm{m} / \mathrm{m}$ cohort, $76 \%$ of cancers in females were aneuploid $(n=19)$ versus $54 \%$ in males $(n=14)$. Despite these differences, there was no statistically significant association between sex and ploidy outcome (diploid versus aneuploid) within genotypic groups ( $b r c a 2 \mathrm{~m} / \mathrm{m} ; \mathrm{tp} 53 \mathrm{~m} / \mathrm{m}$, p $=0.2085 ;$ tp $53 \mathrm{~m} / \mathrm{m}, \mathrm{p}=0.0955$; Chi-square test). However, assessment of the entire study population without segregation by brca 2 genotype revealed that the association between sex and ploidy outcome was statistically significant $(\mathrm{p}=0.0477)$, with aneuploid cancers occurring more frequently in female zebrafish (Table 2).

3.5. brca2 Genotype, Sex, Tumor Ploidy, and Survival Outcome Are Interrelated in Cancer-Bearing Zebrafish. We have previously shown that age at tumor diagnosis is statistically significantly lower in brca $2 \mathrm{~m} / \mathrm{m} ; \mathrm{tp} 53 \mathrm{~m} / \mathrm{m}$ zebrafish compared to $t p 53 \mathrm{~m} / \mathrm{m}$ zebrafish [23]. This finding is consistent with survival outcomes in the current study population, which indicated that brca2 mutation significantly decreases survival time (Figure 3(e), Figures S5A and B, and Table S3). The median age at tumor diagnosis was 8.2 months for brca2 $\mathrm{m} / \mathrm{m} ;$ tp53 $\mathrm{m} / \mathrm{m}$ zebrafish and 10.8 months for tp53 $\mathrm{m} / \mathrm{m}$ zebrafish (Table 1).

Given the difference in ploidy outcomes between male and female zebrafish (Figure 3(d)), we evaluated survival outcomes in male and female cohorts within each genotypic group (Figures 3(f) and 3(g)). Because brca2 genotype significantly impacted age at tumor diagnosis, survival outcomes were assessed only within genotypic groups. In both brca2 $\mathrm{m} / \mathrm{m}$; tp53 $\mathrm{m} / \mathrm{m}$ and tp53 $\mathrm{m} / \mathrm{m}$ zebrafish cohorts, females had a lower median age at tumor diagnosis than males (Table 1), and survival times for females were significantly lower (Figures 3(f) and 3(g), and Table S3).

In human cancers, aneuploidy is often a negative prognostic factor associated with decreased survival time [17-22]. Therefore, we looked for an association between survival time and ploidy outcome for both $\mathrm{brca} 2 \mathrm{~m} / \mathrm{m} ; \mathrm{tp} 53 \mathrm{~m} / \mathrm{m}$ and tp53 $\mathrm{m} / \mathrm{m}$ zebrafish cohorts. In $\mathrm{brca} 2 \mathrm{~m} / \mathrm{m} ; \mathrm{tp} 53 \mathrm{~m} / \mathrm{m}$ zebrafish, the median age at tumor diagnosis was similar for zebrafish with diploid versus aneuploid cancers (Figure 3(h)). In tp53 m/m zebrafish, the median age at tumor diagnosis was lower for 


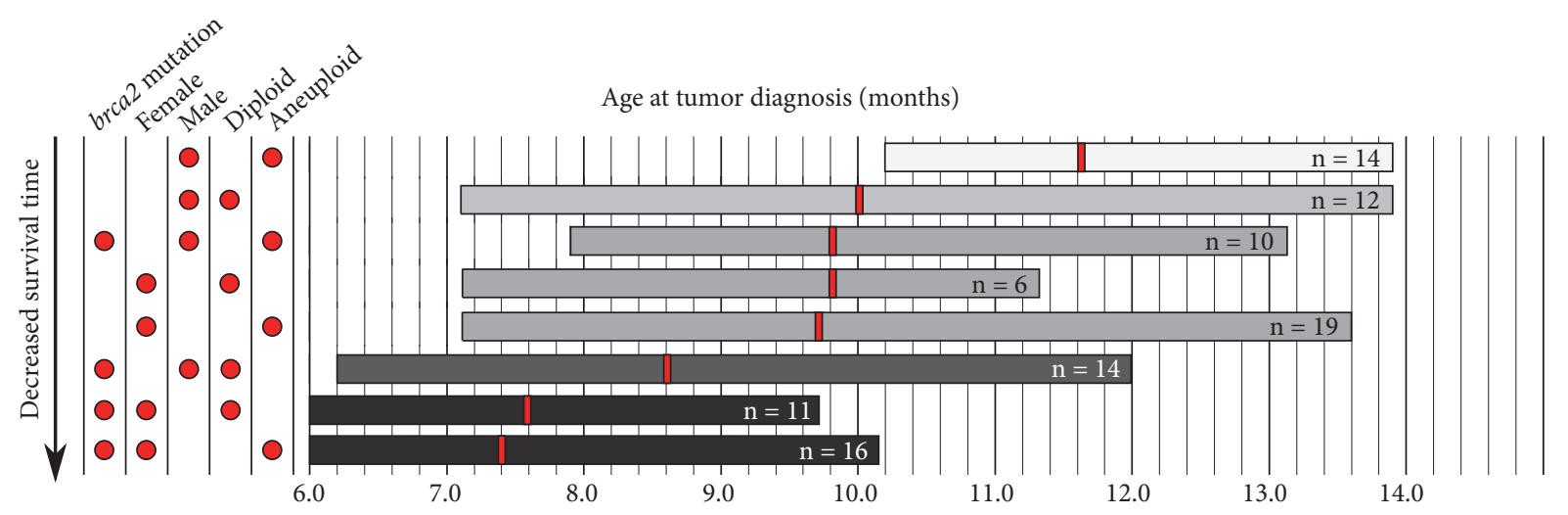

\Median age at tumor diagnosis

Figure 4: Factors contributing to decreased survival time in cancer-bearing zebrafish. Cohorts are represented by horizontal bars and the numbers of individual zebrafish per cohort are indicated. The horizontal width of each bar shows the range of ages at tumor diagnosis within each cohort, and the vertical red line indicates the median age at tumor diagnosis for each cohort. The combination of filled red circles shown to the left of each horizontal bar indicates the defining characteristics of the cohort with regard to brca 2 mutation status, sex, and tumor ploidy. Cohorts are distributed vertically by decreasing survival time, and color range of horizontal bars from light grey to black reflects longest to shortest survival time, respectively.

zebrafish with diploid versus aneuploid cancers (Figure 3(h)). There was no statistically significant difference in survival time for zebrafish with diploid versus aneuploid cancers in either genotypic group (Figures S5C and D and Table S3).

Next, we evaluated for an association between survival time, sex, and ploidy outcome for both brca2 m/m;tp53 m/m and tp53 $\mathrm{m} / \mathrm{m}$ zebrafish cohorts. In both $\mathrm{brca} 2 \mathrm{~m} / \mathrm{m} ; \mathrm{tp} 53$ $\mathrm{m} / \mathrm{m}$ and tp53 $\mathrm{m} / \mathrm{m}$ zebrafish female cohorts, the median age at tumor diagnosis was similar regardless of ploidy status (Figure 3(i)). In contrast, in both $b r c a 2 \mathrm{~m} / \mathrm{m} ; \mathrm{tp} 53 \mathrm{~m} / \mathrm{m}$ and tp53 $\mathrm{m} / \mathrm{m}$ zebrafish male cohorts, the median age at tumor diagnosis was lower for male zebrafish with diploid cancers (Figure 3(i)). We evaluated survival time among individuals of the same sex and genotype that developed diploid versus aneuploid cancers. Within each subgroup of the same genotype and sex, there was no statistically significant difference in survival time based on tumor ploidy (Figures S5E-H and Table S3).

We identified two independent variables, brca2 genotype and sex, that significantly impacted outcomes in this study. Both brca2 mutation and female sex were associated with significantly decreased survival time, and female sex was associated with a significantly increased proportion of aneuploid cancers. Combined evaluation of brca2 genotype, sex, and tumor ploidy did not identify a significant interaction among these three variables (Table S4). However, the three groups with the lowest median ages at tumor diagnosis were defined by a combination of (1) brca2 mutation and female sex (either diploid or aneuploid status) or (2) brca2 mutation and diploid status (either male or female sex) (Figure 4). These data suggest that zebrafish with these combined variables experience a decrease in survival time.

\section{Discussion}

Genomic instability is a hallmark of cancer cells and a critical contributor to the ongoing genetic evolution that accompanies malignant progression. Chromosomal instability (CIN) is one of the most common forms of genomic instability identified in cancer cells and contributes to the development of both structural aberrations (e.g., rearrangements, amplifications, and deletions) and numerical aberrations (aneuploidy) [37, 38]. Aneuploidy can be an indication of ongoing CIN in cancer cells $[39,40]$ and is often a negative prognostic indicator in humans with cancer [1722]. However, this may not be a universal paradigm [16]. Aneuploidy may represent a stable state in cancer cells and does not necessarily indicate ongoing CIN [22, 40-43]. It has also been proposed that aneuploidy may itself induce $\mathrm{CIN}$ and thus contribute to a progressively greater level of aneuploidy in cancers $[44,45]$. Thus, the development of aneuploidy in cancer cells may be both a cause and consequence of genomic instability in cancer.

The tumor suppressor BRCA2 participates in multiple pathways during both meiosis and mitosis that are critical for maintaining genomic integrity, and loss of BRCA2 function can lead to both structural aberrations and aneuploidy [2]. In this study, we used a brca2-mutant/tp53-mutant zebrafish line to investigate the impact of brca2 mutation on cell cycle progression and ploidy outcome in normal tissue (testicular germ cells and somatic cells) and cancers. These heritable brca 2 and $t p 53$ mutations in zebrafish are similar in location and type to pathologic BRCA2 and TP53 mutations in humans $[23,29]$. We have previously demonstrated genetic similarities between BRCA2-associated human and zebrafish cancers, such as the collaborative effect of TP53 mutation on carcinogenesis and the loss of heterozygosity in cancer cells $[23,30]$.

Given BRCA2's role in the resolution of DNA breaks generated during prophase I of meiosis I $[3,4]$, we first assessed nonneoplastic germ cells from adult zebrafish. As the large size of zebrafish oocytes precludes analysis by flow cytometry, we focused these studies on zebrafish testes. We and others have shown that Brca2 is expressed in 
spermatogonia and spermatocytes in vertebrate testes [3, 23, 46] and that testes from Brca2-mutant animals exhibit arrested spermatogenesis $[3,4,23]$. In the current study, testes from brca $2 \mathrm{~m} / \mathrm{m}$ zebrafish showed an accumulation of cells with 4C DNA content, indicating arrest in meiosis I before completion of the first meiotic cell division. These findings are consistent with previous studies of meiotic progression in Brca2-deficient mouse testes [3, 4]. We identified a significantly increased proportion of cells in S-phase from brca2-mutant testes. This finding could reflect cell cycle delay; alternatively, it is possible that some cells categorized as Sphase were aneuploid, with DNA content between $2 \mathrm{C}$ and 4C. Additional studies will be required to distinguish between these possibilities.

Studies in Tp53-deficient mouse models and Drosophila have identified a physiologic function for $\mathrm{p} 53$ during mitosis and meiosis in gonads [47-50]. In mammalian and zebrafish testes, the mitotic phase of spermatogenesis encompasses development of type A and type B spermatogonia, with type A representing a less differentiated population than type B $[33,36]$. These mitotic germ cells undergo both proliferation and differentiation before entering meiosis I as preleptotene spermatocytes [51]. In Tp53-deficient mice, type A spermatogonia are significantly increased [47]; spermatogonia are also increased in tp53-deficient Drosophila [48]. Similarly, we observed that $t p 53 \mathrm{~m} / \mathrm{m}$ zebrafish testes exhibited significant expansion of the type A spermatogonial population, which corresponded to a significant increase in the proportion of cells with 2C DNA content. Spermatogonial expansion in Tp53-deficient mice and Drosophila was attributed to the loss of p53-dependent programmed cell death in mitotic germ cells $[47,48]$. p53 was also expressed during meiotic recombination in mouse and Drosophila germ cells following the induction of double-strand DNA breaks by the topoisomerase Spol1 [47, 50]. However, loss of p53 does not appear to alter meiotic progression in germ cells in the absence of additional stimuli (e.g., ionizing radiation) $[47,50]$, although meiotic recombination frequency is reduced [50]. Similarly, our data suggests that loss of p53 does not alter meiotic progression in zebrafish testes: the proportions of cells in 1C, $4 \mathrm{C}$, and S-phase compartments were equivalent in $t p 53 \mathrm{~m} / \mathrm{m}$ testes compared to wild type testes, and tp53 $\mathrm{m} / \mathrm{m}$ testes were histologically normal.

Strikingly, combined mutations in brca2 and tp53 resulted in meiotic arrest and a dramatic accumulation of cells with $2 \mathrm{C}$ DNA content, correlating to significantly increased numbers of both type A and type B spermatogonia. These data indicate the significant expansion of mitotic germ cells in brca2 $\mathrm{m} / \mathrm{m} ; \mathrm{tp} 53 \mathrm{~m} / \mathrm{m}$ zebrafish testes. The predominance of type A spermatogonia, which are less differentiated than type B spermatogonia, suggests that spermatogonial differentiation is suppressed in brca $2 \mathrm{~m} / \mathrm{m} ; t p 53 \mathrm{~m} / \mathrm{m}$ zebrafish testes. These outcomes are distinct from the effects of brca2 or tp53 mutations alone in zebrafish testes, which caused meiotic arrest or selective type A spermatogonial expansion, respectively. We have not identified a similar effect in published studies of Brca2-mutant;Tp53-mutant mouse models; a synergistic suppressive effect on germ cell expansion during the initiation of meiosis has been described in testes from juvenile mice with combined mutations in Brca2 and Palb2 [4]. However, combined mutations in tp53 and rad54 have been shown to alter germ cell numbers in Drosophila ovary [50]. Rad54 functions downstream of Brca2 and is essential for homology-directed recombination and DNA repair [52]. Drosophila ovaries with combined tp53 and rad54 mutations showed a variable, frequently increased number of mitotic germ cells (known as nurse cells) [50], which is comparable to the outcome we observed in $b r c a 2 \mathrm{~m} / \mathrm{m} ; \mathrm{tp} 53 \mathrm{~m} / \mathrm{m}$ zebrafish testes.

The above-described effects of combined brca2 and tp53 mutations on mitotic germ cells (spermatogonia) in zebrafish suggest the interesting possibility that concurrent mutations in BRCA2 and TP53 could synergistically promote proliferation and suppress differentiation, which has important implications in the context of cancer initiation. Spermatogenesis is considered to be a classical stem cell-driven process, providing a model for analyzing stem cell physiology and behavior that may be applicable to stem cell populations in other tissues $[53,54]$. It is possible that tissue stem and progenitor cells in other sites, which are potential sources for the emergence of cancer stem cells, may be similarly affected by combined BRCA2 and TP53 mutations. In support of this concept, BRCA1 mutation or knockdown has been linked to increased stem/progenitor cell populations and dedifferentiation of stem cells in human breast and mouse mammary tissues $[55,56]$. Also, women with BRCA1- or BRCA2associated cancer had an increased frequency of breast stem cells in noncancerous breast tissue, which were identified by expression of the stem and progenitor cell marker ALDH [57]. P53 has a known role in the maintenance and regulation of both embryonic and adult stem cells, and wild type p53 suppresses self-renewal and induces differentiation of stem cells after DNA damage (reviewed by Aloni-Grinstein R et al.) [58]. Additionally, proliferation is increased in p53-deficient stem and progenitor cells [59-61]. Together these studies suggest that further investigation of a potentially synergistic role for BRCA2 and TP53 mutations in disrupting stem and progenitor cell homeostasis may provide new insight into how mutations in these genes modulate carcinogenesis.

Next, we assessed nonneoplastic somatic tissues and cancers from adult zebrafish. DNA content analysis of nonneoplastic somatic tissues from zebrafish indicated that brca2 mutation does not alter ploidy in these cells. This outcome is similar to what has been observed in mammalian cells, namely, that normal, nonneoplastic cells generally do not tolerate aneuploidy [37, 43, 44, 62]. We identified a small percentage of aneuploid cells in four somatic tissue samples but cannot rule out the possibility that these tissues contained early-stage cancers not detectable by stereomicroscopic examination. In zebrafish cancers, we observed that diploidy was more common in brca2-associated cancers than non-brca2-associated cancers, although this difference was not statistically significant. In comparison, diploid and aneuploid cancers reportedly occur in roughly similar proportions in human BRCA2-associated and non-BRCA2-associated cancers [14-16]. Contrastingly, aneuploidy and polyploidy were increased in Brca2-inactivated tumor cell lines derived from a mouse model of pancreatic ductular adenocarcinoma 
[63]. Overall, our data from brca2-associated zebrafish cancers parallels previous reports that BRCA2 mutation does not significantly increase the rate of aneuploidy in human cancers.

We additionally considered sex as a variable that might influence tumor ploidy in zebrafish. Aneuploidy was significantly more common in female zebrafish in the full study population, although significance was not maintained when study cohorts were segregated by brca2 genotype. To our knowledge, the impact of sex on tumor ploidy has not been previously reported in zebrafish cancer models. In humans, gender is not linked to the development of global numerical aberrations in cancers, although numerical aberrations specifically affecting the sex chromosomes (gonosomes) occur more frequently in cancers from males [64]. However, gender-specific structural aberrations affecting both gonosomes and autosomes are reported in some cancer types that may be biologically and prognostically significant [6467]. The factors that drive accumulation of these genderassociated genomic changes are not yet defined. Our zebrafish model will be an informative tool for investigating how sex impacts the accumulation of genetic and genomic changes during carcinogenesis.

Finally, we evaluated survival outcome in cancer-bearing zebrafish in the context of the three major variables analyzed in this study (brca2 genotype, sex, and tumor ploidy). We have previously reported that brca2-mutant zebrafish develop tumors at a significantly younger age than non-brca2-mutant zebrafish [23], which was also observed in this study. We additionally identified a significant impact of sex on survival outcome: females in both genotypic groups developed tumors at a statistically significant younger age than males. We are unaware of any previous report of a zebrafish cancer model that experiences a significant disparity in survival outcome based on sex. In humans, survival outcomes in females are generally better than in males [68]. However, there are some cancer types for which survival outcomes are reversed; i.e. survival outcomes in women are worse than in men $[69,70]$; such differences have also been linked to response to targeted therapies [71]. Gender-associated differences in survival outcome have yet to be explained in humans, although multiple possible contributors, including hormonal signaling, environmental exposures, DNA repair defects, and other factors, have been postulated $[65,70]$. The potential contributions of such factors to carcinogenesis in our zebrafish model are not yet known.

Ploidy is an independent prognostic factor for survival across a variety of human cancer types, with aneuploidy associated with worse prognosis [17-22]. Within both genotypic groups of cancer-bearing zebrafish in this study, we found that median survival times in females with diploid versus aneuploid cancers were similar, while median survival times for males with diploid cancers were lower than for males with aneuploid cancers. This finding is surprising, given that aneuploidy is generally linked to worse prognosis in human cancer patients. However, diploidy has been correlated to worse prognosis in BRCA2-associated human breast cancers [16]. In our study, ploidy did not emerge as a variable that significantly contributed to survival outcome in cancer-bearing zebrafish. Since we identified both brca2 genotype and sex as variables that significantly influenced survival outcome, we could not assess the impact of ploidy on survival outcome independently from these variables. As a result, we cannot rule out the possibility that ploidy would have been found to significantly affect survival outcome in a larger study population. In comparison, the aforementioned study of ploidy status and survival outcome in human breast cancer patients with and without BRCA2 mutation presented data from almost 3,000 patients that was acquired over a 50year period [16].

We observed the lowest median survival times in zebrafish with (1) brca2 mutation and female sex and (2) brca2 mutation and diploid cancer (Figure 4). Although diploidy is typically linked to relative genomic stability, diploid cancers may actually be "pseudodiploid," exhibiting complex genomic alterations that do not impact total chromosomal content. This condition has been described in diploid BRCA2associated human cancers [15] and was proposed as a contributor to poor prognosis in patients with diploid BRCA2associated cancers [16]. Similarly, near-diploid colorectal cancers have been shown to possess extensive genomic changes that may be essential in carcinogenesis [72]. On the other hand, not all aneuploid cancers experience ongoing CIN but rather exhibit relative genomic stability [22, 40-43]. It is therefore possible that, in our model system, diploid or aneuploid categorizations do not reflect the level of genomic stability in these zebrafish cancers. Further studies are underway to investigate more deeply the genetic and genomic alterations that characterize cancers in our zebrafish model and determine the impact of brca2 mutation and sex on these alterations.

\section{Conclusions}

Our findings confirm that the individual effects of brca2 and tp53 mutations on testicular germ cell development are conserved in zebrafish and reveal that combined brca 2 and tp53 mutations collaborate to promote accumulation of spermatogonia while suppressing spermatogonial differentiation. Our findings additionally identify both brca2 genotype and sex as independent variables that significantly affect survival outcome in cancer-bearing zebrafish. While ploidy outcome in zebrafish cancers did not significantly affect survival outcome, ploidy was significantly influenced by sex. Finally, we determined that diploidy is not linked to better survival outcome in cancer-bearing zebrafish: the worst survival outcomes were observed with (1) brca2 mutation and female sex and (2) brca2 mutation and diploid cancer. These studies provide new insight into the impact of combined BRCA2 and TP53 mutations on germ cell development and identify key influences of BRCA2 mutation, sex, and ploidy on survival outcome in vertebrate cancer.

\section{Data Availability}

All data generated or analyzed during this study are included in this published article (and its Supplementary Information files). The zebrafish lines described in this work (wild type 
(AB), brca $2^{\text {hg5 }}$ mutant, and $t p 53^{z d f 1}$ mutant) are maintained at the Shive laboratory and may be accessed by contacting the corresponding author.

\section{Conflicts of Interest}

The authors declare no conflicts of interest.

\section{Authors' Contributions}

H. R. Shive conceived and designed the experiments. L. Mensah, J. L. Ferguson, and H. R. Shive performed the experiments. H. R. Shive analyzed and interpreted the data and prepared figures. L. Mensah, J. L. Ferguson, and H. R. Shive wrote the paper.

\section{Acknowledgments}

This work was supported by NIH K01OD021419 and by NC State University, College of Veterinary Medicine.

\section{Supplementary Materials}

The supplementary materials include Supplementary Figure Legends, Supplementary Figures S1-S5, and Supplementary Tables S1-S4. Presented in the Supplementary Figures are the following data: Figure S1, gating strategies for analysis of flow cytometry data from testes, nonneoplastic somatic cells, and cancer cells; Figure S2, representative histologic images of testes from brca2 m/m;tp53 m/m and tp53 m/m zebrafish; Figure S3, distributions of cells (mean percent of gated cells) according to DNA content in nonneoplastic somatic tissues and cancers; Figure S4, example of disparate ploidy outcomes from two anatomically distinct cancers derived from the same zebrafish; and, Figure S5, analysis of survival outcome with respect to various population characteristics ( brca2 mutation status, sex, and tumor ploidy). Presented in the Supplementary Tables are the following data: Table S1, comparison of DNA content and spermatogonia counts in testes from wild type, tp53 $\mathrm{m} / \mathrm{m} ; \operatorname{brca} 2 \mathrm{~m} / \mathrm{m}$, and brca $2 \mathrm{~m} / \mathrm{m} ; \mathrm{tp} 53 \mathrm{~m} / \mathrm{m}$ zebrafish; Table S2, definition of DNA ploidy categories determined by the calculated DNA index; Table S3, summary of statistical analyses of survival outcomes in cancer-bearing zebrafish; and, Table S4, summary of statistical analyses assessing the impact of multiple variables on survival outcomes in cancer-bearing zebrafish. (Supplementary Materials)

\section{References}

[1] E. Duro and A. L. Marston, "From equator to pole: splitting chromosomes in mitosis and meiosis," Genes \& Development, vol. 29, pp. 109-122, 2015.

[2] A. R. Venkitaraman, "Tumour suppressor mechanisms in the control of chromosome stability: Insights from BRCA2," Molecules and Cells, vol. 37, no. 2, pp. 95-99, 2014.

[3] S. K. Sharan, A. Pyle, V. Coppola et al., "BRCA2 deficiency in mice leads to meiotic impairment and infertility," Development, vol. 131, no. 1, pp. 131-142, 2004.
[4] S. A. Hartford, R. Chittela, X. Ding et al., "Interaction with PALB2 is essential for maintenance of genomic integrity by BRCA2," PLoS Genetics, vol. 12, no. 8, p. e1006236, 2016.

[5] K. Seeliger, S. Dukowic-Schulze, R. Wurz-Wildersinn et al., "BRCA2 is a mediator of RAD51- and DMC1-facilitated homologous recombination in Arabidopsis thaliana," New Phytologist, vol. 193, no. 2, pp. 364-375, 2012.

[6] K. J. Patel, V. P. Yu, H. Lee et al., "Involvement of Brca2 in DNA repair," Molecular Cell, vol. 1, no. 3, pp. 347-357, 1998.

[7] H. Lee, A. H. Trainer, L. S. Friedman et al., "Mitotic checkpoint inactivation fosters transformation in cells lacking the breast cancer susceptibility gene, Brca2," Molecular Cell, vol. 4, no. 1, pp. 1-10, 1999.

[8] E. Choi, P.-G. Park, H.-O. Lee et al., "BRCA2 fine-tunes the spindle assembly checkpoint through reinforcement of BubR1 acetylation," Developmental Cell, vol. 22, no. 2, pp. 295-308, 2012.

[9] M. J. Daniels, Y. Wang, M. Lee, and A. R. Venkitaraman, "Abnormal cytokinesis in cells deficient in the breast cancer susceptibility protein BRCA2," Science, vol. 306, no. 5697, pp. 876-879, 2004.

[10] M. Lee, M. J. Daniels, M. J. Garnett, and A. R. Venkitaraman, "A mitotic function for the high-mobility group protein HMG20b regulated by its interaction with the BRC repeats of the BRCA2 tumor suppressor," Oncogene, vol. 30, no. 30, pp. 3360-3369, 2011.

[11] N. Ayoub, E. Rajendra, X. Su, A. D. Jeyasekharan, R. Mahen, and A. R. Venkitaraman, "The carboxyl terminus of Brca2 links the disassembly of Rad51 complexes to mitotic entry," Current Biology, vol. 19, no. 13, pp. 1075-1085, 2009.

[12] T. Menzel, V. Nähse-Kumpf, A. N. Kousholt et al., "A genetic screen identifies BRCA2 and PALB2 as key regulators of G2 checkpoint maintenance," EMBO Reports, vol. 12, no. 7, pp. 705712, 2011.

[13] K. Schlacher, N. Christ, N. Siaud, A. Egashira, H. Wu, and M. Jasin, "Double-strand break repair-independent role for BRCA2 in blocking stalled replication fork degradation by MRE11," Cell, vol. 145, no. 4, pp. 529-542, 2011.

[14] A. B. Jonsdottir, O. A. Stefansson, J. Bjornsson, J. G. Jonasson, H. M. Ogmundsdottir, and J. E. Eyfjord, "Tetraploidy in BRCA2 breast tumours," European Journal of Cancer, vol. 48, no. 3, pp. 305-310, 2012.

[15] O. A. Stefansson, J. G. Jonasson, K. Olafsdottir et al., "Genomic and phenotypic analysis of BRCA2 mutated breast cancers reveals co-occurring changes linked to progression," Breast Cancer Research, vol. 13, p. R95, 2011.

[16] L. Tryggvadottir, E. J. Olafsdottir, G. H. Olafsdottir et al., "Tumour diploidy and survival in breast cancer patients with BRCA2 mutations," Breast Cancer Research and Treatment, vol. 140, no. 2, pp. 375-384, 2013.

[17] O. P. Kallioniemi, G. Blanco, M. Alavaikko et al., “Tumour DNA ploidy as an independent prognostic factor in breast cancer," British Journal of Cancer, vol. 56, no. 5, pp. 637-642, 1987.

[18] K. Herman, A. Gruchala, A. Niezabitowski et al., "Prognostic factors in retroperitoneal sarcomas: ploidy of DNA as a predictor of clinical outcome," Journal of Surgical Oncology, vol. 71, pp. 32-35, 1999.

[19] A. E. Pinto, S. Andre, and J. Soares, "Short-term significance of DNA ploidy and cell proliferation in breast carcinoma: a multivariate analysis of prognostic markers in a series of 308 patients," Journal of Clinical Pathology, vol. 52, pp. 604-611, 1999. 
[20] P. San Miguel-Fraile, R. Carrillo-Gijon, J. L. Rodriguez-Peralto, and I. A. Badiola, "Prognostic significance of DNA ploidy and proliferative index (MIB-1 index) in childhood rhabdomyosarcoma," American Journal of Clinical Pathology, vol. 121, pp. 358$365,2004$.

[21] D. Choma, J. P. Daures, X. Quantin, and J. L. Pujol, “Aneuploidy and prognosis of non-small-cell lung cancer: a meta-analysis of published data," British Journal of Cancer, vol. 85, pp. 14-22, 2001.

[22] L. M. Zasadil, E. M. Britigan, and B. A. Weaver, "2n or not 2n: Aneuploidy, polyploidy and chromosomal instability in primary and tumor cells," Seminars in Cell and Developmental Biology, vol. 24, pp. 370-379, 2013.

[23] H. R. Shive, R. R. West, L. J. Embree et al., "brca2 in zebrafish ovarian development, spermatogenesis, and tumorigenesis," Proceedings of the National Acadamy of Sciences of the United States of America, vol. 107, no. 45, pp. 19350-19355, 2010.

[24] B. Evers and J. Jonkers, "Mouse models of BRCA1 and BRCA2 deficiency: past lessons, current understanding and future prospects," Oncogene, vol. 25, pp. 5885-5897, 2006.

[25] T. Crook, L. A. Brooks, S. Crossland et al., "p53 mutation with frequent novel condons but not a mutator phenotype in BRCA1and BRCA2-associated breast tumours," Oncogene, vol. 17, pp. 1681-1689, 1998.

[26] S. J. Ramus, L. G. Bobrow, P. D. Pharoah et al., "Increased frequency of TP53 mutations in BRCA1 and BRCA2 ovarian tumours," Genes, Chromosomes and Cancer, vol. 25, no. 2, pp. 91-96, 1999.

[27] R. Roy, J. Chun, and S. N. Powell, "BRCA1 and BRCA2: different roles in a common pathway of genome protection," Nature Reviews Cancer, vol. 12, pp. 68-78, 2011.

[28] S. Gretarsdottir, S. Thorlacius, R. Valgardsdottir et al., "BRCA2 and p53 mutations in primary breast cancer in relation to genetic instability," Cancer Research, vol. 58, no. 5, pp. 859-862, 1998.

[29] S. Berghmans, R. D. Murphey, E. Wienholds et al., "tp53 mutant zebrafish develop malignant peripheral nerve sheath tumors," Proceedings of the National Acadamy of Sciences of the United States of America, vol. 102, no. 2, pp. 407-412, 2005.

[30] H. R. Shive, R. R. West, L. J. Embree et al., "BRCA2 and TP53 collaborate in tumorigenesis in zebrafish," PLoS One, vol. 9, article e87177, 2014.

[31] L. A. White, J. M. Sexton, and H. R. Shive, "Histologic and immunohistochemical analyses of soft tissue sarcomas From brca2-mutant/tp53-mutant zebrafish are consistent with neural crest (schwann cell) origin," Veterinary Pathology, vol. 54, no. 2, pp. 320-327, 2017.

[32] J. Schindelin, I. Arganda-Carreras, and E. Frise, "Fiji: an opensource platform for biological-image analysis," Nature Methods, vol. 9, no. 7, pp. 676-682, 2012.

[33] M. C. Leal, E. R. Cardoso, R. H. Nóbrega et al., "Histological and stereological evaluation of zebrafish (Danio rerio) spermatogenesis with an emphasis on spermatogonial generations," Biology of Reproduction, vol. 81, no. 1, pp. 177-187, 2009.

[34] E. Rotgers, S. Cisneros-Montalvo, K. Jahnukainen, J. Sandholm, J. Toppari, and M. Nurmio, "A detailed protocol for a rapid analysis of testicular cell populations using flow cytometry," Andrology, vol. 3, no. 5, pp. 947-955, 2015.

[35] J. S. Ross, G. P. Linette, J. Stec, M. S. Ross, S. Anwar, and A. Boguniewicz, "DNA ploidy and cell cycle analysis in breast cancer," American Journal of Clinical Pathology, vol. 120, no. suppl_1, pp. S72-S84, 2003.
[36] E. C. Roosen-Runge and L. O. Giesel Jr., "Quantitative studies on spermatogenesis in the albino rat," American Journal of Anatomy, vol. 87, Article ID 1000870102, pp. 1-30, 1950.

[37] L. C. Funk, L. M. Zasadil, and B. A. Weaver, "Living in CIN: mitotic infidelity and its consequences for tumor promotion and suppression," Developmental Cell, vol. 39, pp. 638-652, 2016.

[38] S. Negrini, V. G. Gorgoulis, and T. D. Halazonetis, "Genomic instability-an evolving hallmark of cancer," Nature Reviews Molecular Cell Biology, vol. 11, pp. 220-228, 2010.

[39] S. L. Thompson, S. F. Bakhoum, and D. A. Compton, "Mechanisms of chromosomal instability," Current Biology, vol. 20, pp. R285-R295, 2010.

[40] T. A. Potapova, J. Zhu, and R. Li, "Aneuploidy and chromosomal instability: a vicious cycle driving cellular evolution and cancer genome chaos," Cancer and Metastasis Reviews, vol. 32, pp. 377389,2013

[41] D. J. Gordon, B. Resio, and D. Pellman, "Causes and consequences of aneuploidy in cancer," Nature Reviews Genetics, vol. 13, pp. 189-203, 2012.

[42] Z. Storchova and C. Kuffer, "The consequences of tetraploidy and aneuploidy," Journal of Cell Science, vol. 121, pp. 3859-3866, 2008.

[43] R. H. van Jaarsveld and G. Kops, "Difference makers: chromosomal instability versus aneuploidy in cancer," Trends in Cancer, vol. 2, pp. 561-571, 2016.

[44] S. Santaguida and A. Amon, "Short- and long-term effects of chromosome mis-segregation and aneuploidy," Nature Reviews Molecular Cell Biology, vol. 16, pp. 473-485, 2015.

[45] M. Giam and G. Rancati, "Aneuploidy and chromosomal instability in cancer: a jackpot to chaos," Cell Division, vol. 10, article 3, 2015.

[46] P. E. Blackshear, S. M. Goldsworthy, J. F. Foley et al., "Brcal and Brca2 expression patterns in mitotic and meiotic cells of mice," Oncogene, vol. 16, no. 1, pp. 61-68, 1998.

[47] T. L. Beumer, H. L. Roepers-Gajadien, I. S. Gademan et al., "The role of the tumor suppressor p53 in spermatogenesis," Cell Death \& Differentiation, vol. 5, no. 8, pp. 669-677, 1998.

[48] F. Napoletano, B. Gibert, K. Yacobi-Sharon et al., "p53-dependent programmed necrosis controls germ cell homeostasis during spermatogenesis," PLoS Genetics, vol. 13, article e1007024, 2017.

[49] M. Xiong, I. C. Ferder, Y. Ohguchi, and N. Wang, "Quantitative analysis of male germline stem cell differentiation reveals a role for the p53-mTORC1 pathway in spermatogonial maintenance," Cell Cycle, vol. 14, no. 18, pp. 2905-2913, 2015.

[50] W. J. Lu, J. Chapo, I. Roig, and J. M. Abrams, "Meiotic recombination provokes functional activation of the p53 regulatory network," Science, vol. 328, no. 5983, pp. 1278-1281, 2010.

[51] P. P. Lie, C. Y. Cheng, and D. D. Mruk, "Coordinating cellular events during spermatogenesis: a biochemical model," Trends in Biochemical Sciences, vol. 34, pp. 366-373, 2009.

[52] A. V. Mazin, O. M. Mazina, D. V. Bugreev, and M. J. Rossi, "Rad54, the motor of homologous recombination," DNA Repair, vol. 9, pp. 286-302, 2009.

[53] J. M. Oatley and R. L. Brinster, "Regulation of spermatogonial stem cell self-renewal in mammals," Annual Review of Cell and Developmental Biology, vol. 24, pp. 263-286, 2008.

[54] S. Yoshida, "Elucidating the identity and behavior of spermatogenic stem cells in the mouse testis," Reproduction, vol. 144, no. 3, pp. 293-302, 2012. 
[55] F. Bai, H. L. Chan, A. Scott et al., "BRCA1 suppresses epithelialto-mesenchymal transition and stem cell dedifferentiation during mammary and tumor development," Cancer Research, vol. 74, no. 21, pp. 6161-6172, 2014.

[56] S. Liu, C. Ginestier, E. Charafe-Jauffret et al., "BRCA1 regulates human mammary stem/progenitor cell fate," Proceedings of the National Academy of Sciences of the United States of America, vol. 105, pp. 1680-1685, 2008.

[57] B. L. Isfoss, B. Holmqvist, H. Jernstrom, P. Alm, and H. Olsson, "Women with familial risk for breast cancer have an increased frequency of aldehyde dehydrogenase expressing cells in breast ductules," BMC Clinical Pathology, vol. 13, pp. 13-28, 2013.

[58] R. Aloni-Grinstein, Y. Shetzer, T. Kaufman, and V. Rotter, "p53: the barrier to cancer stem cell formation," FEBS Lett588, pp. 2580-2589, 2014.

[59] A. Armesilla-Diaz, G. Elvira, and A. Silva, "p53 regulates the proliferation, differentiation and spontaneous transformation of mesenchymal stem cells," Experimental Cell Research, vol. 315, pp. 3598-3610, 2009.

[60] H. Liu, D. Jia, A. Li et al., "P53 regulates neural stem cell proliferation and differentiation via bmp-smad1 signaling and ID1," Stem Cells and Development, vol. 22, no. 6, pp. 913-927, 2013.

[61] A. M. McConnell, C. Yao, A. R. Yeckes et al., "p53 regulates progenitor cell quiescence and differentiation in the airway," Cell Reports, vol. 17, no. 9, pp. 2173-2182, 2016.

[62] K. Tanaka and T. Hirota, "Chromosomal instability: A common feature and a therapeutic target of cancer," Biochim Biophys Acta, vol. 1866, pp. 64-75, 2016.

[63] M. Rowley, A. Ohashi, G. Mondal et al., "Inactivation of Brca2 promotes Trp53-associated but inhibits KrasG12D-dependent pancreatic cancer development in mice," Gastroenterology, vol. 140, no. 4, pp. 1303-1313, 2011.

[64] S. Schulze and I. Petersen, "Gender and ploidy in cancer survival," Cellular oncology (Dordrecht), vol. 34, pp. 199-208, 2011.

[65] J. Zhang, S. Yan, X. Liu et al., "Gender-related prognostic value and genomic pattern of intra-tumor heterogeneity in colorectal cancer," Carcinogenesis, vol. 38, no. 8, pp. 837-846, 2017.

[66] R. H. Ali, M. J. Marafie, M. S. Bitar et al., "Gender-associated genomic differences in colorectal cancer: Clinical insight from feminization of male cancer cells," International Journal of Molecular Sciences, vol. 15, no. 10, pp. 17344-17365, 2014.

[67] L. Bottarelli, C. Azzoni, F. Necchi et al., "Sex chromosome alterations associate with tumor progression in sporadic colorectal carcinomas," Clinical Cancer Research, vol. 13, no. 15, pp. 43654370, 2007.

[68] M. B. Cook, K. A. McGlynn, S. S. Devesa et al., "Sex disparities in cancer mortality and survival," Cancer Epidemiology, Biomarkers \& Prevention, vol. 20, pp. 1629-1637, 2011.

[69] H. A. Risch, G. R. Howe, M. Jain et al., "Are female smokers at higher risk for lung cancer than male smokers? A case-control analysis by histologic type," American Journal of Epidemiology, vol. 138, no. 1-2, pp. 281-293, 1993.

[70] J. Dobruch, S. Daneshmand, M. Fisch et al., "Gender and bladder cancer: a collaborative review of etiology, biology, and outcomes," European Urology, vol. 69, no. 2, pp. 300-310, 2016.

[71] J. A. Pinto, C. S. Vallejos, L. E. Raez et al., "Gender and outcomes in non-small cell lung cancer: an old prognostic variable comes back for targeted therapy and immunotherapy?" ESMO Open, vol. 3, article e000344, 2018.
[72] M. Muleris, A. Chalastanis, N. Meyer et al., "Chromosomal instability in near-diploid colorectal cancer: a link between numbers and structure," PLoS ONE, vol. 3, article e1632, 2008. 


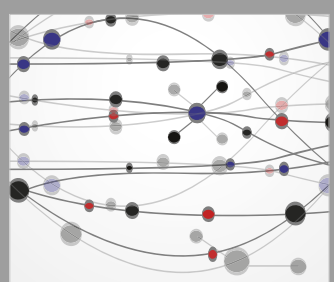

The Scientific World Journal
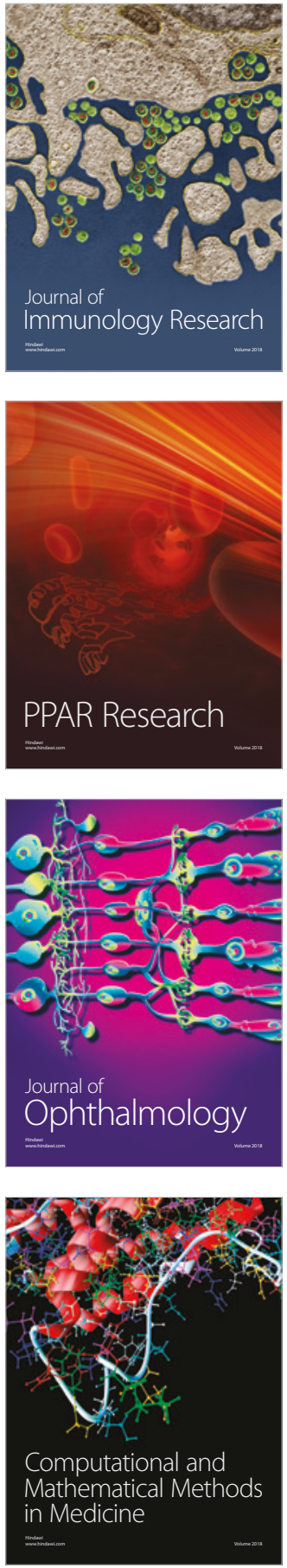

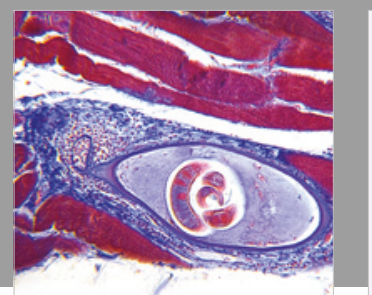

Gastroenterology Research and Practice

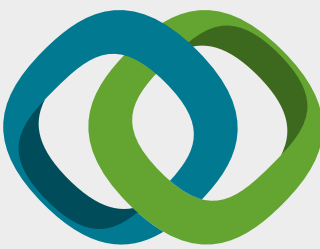

\section{Hindawi}

Submit your manuscripts at

www.hindawi.com
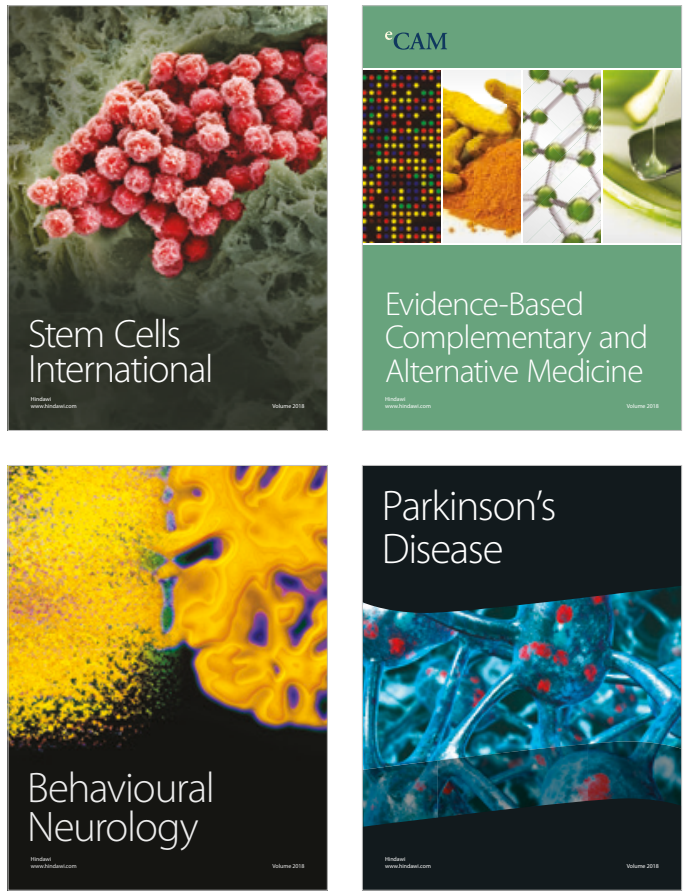

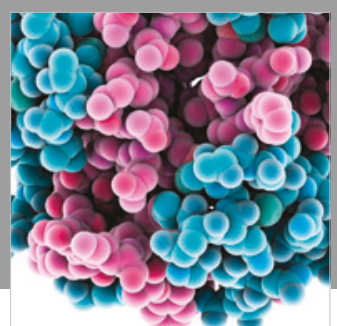

ournal of

Diabetes Research

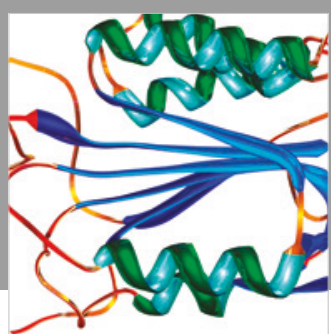

Disease Markers
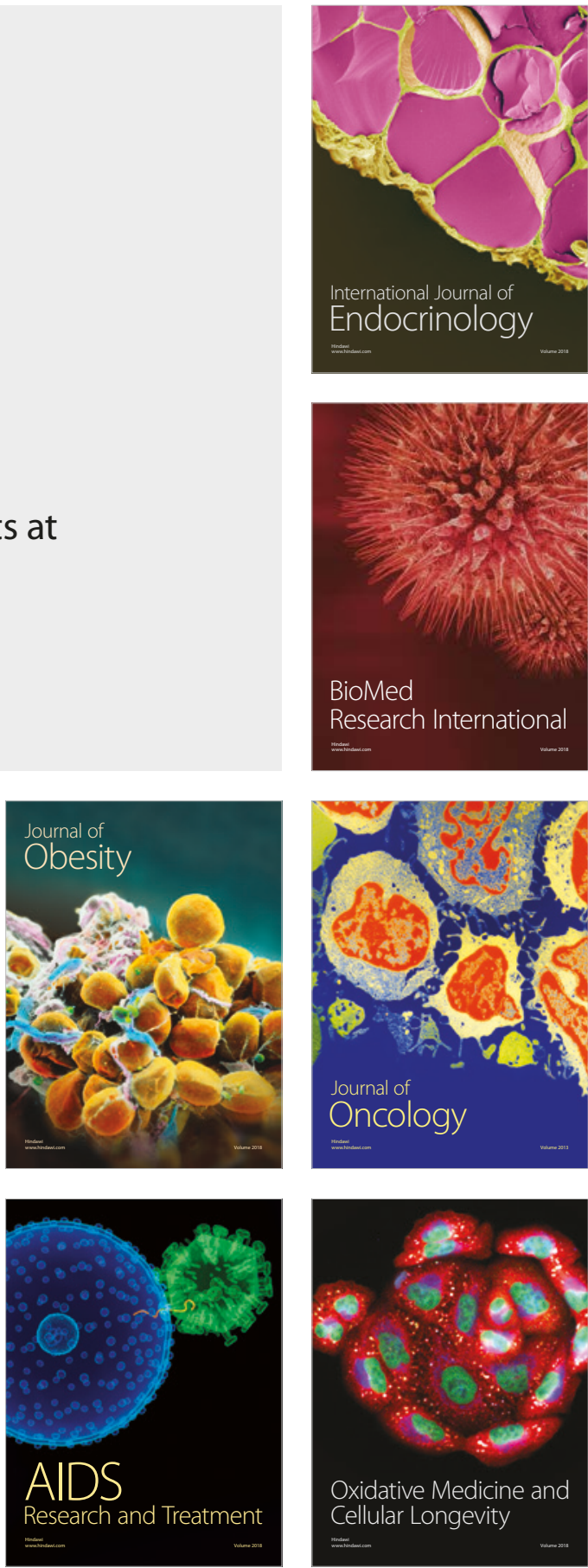